\title{
Charting Hydrogen Bond Anisotropy
}

\author{
Diogo Santos-Martins ${ }^{1}$ and Stefano Forli, ${ }^{1, \mathbb{}}$ \\ ${ }^{1}$ Department of Integrative Structural and Computational Biology, The Scripps Research Institute, La Jolla, 92037, California, USA
}

\begin{abstract}
Hydrogen bond (HB) is an essential interaction in countless phenomena, and regulates the chemistry of life. HBs are characterized by two main features, strength and directionality, with a high degree of heterogeneity across different chemical groups. These characteristics are dependent on the electronic configuration of the atoms involved in the interaction, which, in turn, is influenced strongly by the molecular environment where they are found. Studies based on the analysis of HB in solid phase, such as X-ray crystallography, suffer from significant biases due to the packing forces. These will tend to better describe strong HBs at the expenses of weak ones, which are either distorted or under-represented. Using quantum mechanics (QM), we calculated interaction energies for about a hundred acceptor and donors, in a rigorously defined set of geometries. We performed about 180,000 independent $Q M$ calculations, covering all relevant angular components, and mapping strength and directionality in a context free from external biases, with both single-site and cooperative HBs. We show that by quantifying directionality, there is not correlation with strength, and therefore these two components need to be addressed separately. Results demonstrate that there are very strong $\mathrm{HB}$ acceptors (e.g., DMSO) with nearly isotropic interactions, and weak ones (e.g., thioacetone) with a sharp directional profile. Similarly, groups can have comparable directional propensity, but be very distant in the strength spectrum (e.g., thioacetone and pyridine). These findings have implications for biophysics and molecular recognition, providing new insight for chemical biology, protein engineering, and drug design. The results require rethinking the way directionality is described, with implications for the thermodynamics of $\mathrm{HB}$.
\end{abstract}

hydrogen bond | drug design | force field | non-covalent interaction Correspondence: forli@scripps.edu

\section{Introduction}

Hydrogen bond (HB) plays a role in countless phenomena and has been studied in several fields within chemistry and biology. Arguably, it is the main force that drives reversible interactions in biological contexts ${ }^{1,2}$, being responsible for the physicochemical properties of water ${ }^{3,4}$, orchestrating structural arrangement of proteins ${ }^{5}$, and stabilizing transition states in natural ${ }^{6}$ and engineered catalysts ${ }^{7}$.

The hallmark of HB is its directionality: its strength can be heavily modulated by the geometries of acceptor and donor with respect to each other; the presence of directionality is used to distinguish HB from van der Waals interactions ${ }^{8,9}$. Directionality has been studied by statistical analysis of the Cambridge Structural Database (CSD) ${ }^{10}$ and the Protein Data Bank (PDB) ${ }^{11}$. These analyses are based on the assumption that HB strength and directionality can be inferred from structures because "interaction geometries are conserved with fidelities that correspond to their strengths $" 9,12$. Statistical distributions of such observed geometries can be then translated into chemical potentials ${ }^{13,14}$ Over the past decades, several chemical groups were studied using statistical approaches, including halogens ${ }^{15-17}$, sulfur and oxygen ${ }^{18-20}$, weak and strong donors ${ }^{8}$, among others ${ }^{17,21-25}$

Despite providing invaluable information, retrospective structural analysis has several issues that limit the coverage of HB properties. First, it is chemically inhomogeneous because several chemical groups are under- or overrepresented. Furthermore, structural patterns of HBs can be smeared by factors such as steric hindrance ${ }^{8}$, packing forces ${ }^{26}$, or be biased by the refinement programs used (which often include specific constraints for $\mathrm{HB})^{27}$.

Quantum mechanics (QM) calculations, while requiring more resources than statistical surveys, have been used to characterize various HB properties ${ }^{26,28-35}$, usually with good agreement with observations ${ }^{13}$. Among them, very few calculated interaction energies at non-equilibrium geometries (the ones that did are summarized in Supplementary Table S1). These few studies lack chemical diversity, as relevant chemical groups such as halogens, sulfur or phosphate oxyacids have never been characterized. Furthermore, with respect to the geometrical degrees of freedom, most studies performed one-dimensional scans along a given interaction angle, and only a few considered more than one angle (Supplementary Table S1).

This work is an effort towards a systematic and comprehensive characterization of HB directionality. We have performed about 180,000 energy QM calculations on a broad and diverse panel of chemical groups of relevance in biological and drug design contexts, and performed volumetric energy scans to address multiple angular components, as well as the distance at which the interaction is established. Strength and directionality have been measured and discussed.

\section{Results}

The dataset contains 34 donors (Fig.S1) and 67 acceptors (Fig.S2, Methods). For the entire dataset, calculations were performed using B3LYP density functional with the cc-pVDZ basis set and the counterpoise correction, while MP2/aug-cc-pVTZ with counterpoise correction was used for smaller subsets of complexes. The validation of these two methods, their differences and their effect on the results has been discussed in Supplementary Methods.

Strength. We measured the strength of HBs on the 34 donors (Fig.S1) and 67 acceptors, performing B3LYP/cc-pVDZ calculations of donor-acceptor dimers interacting in optimal ge- 
ometries. Interaction energies $(\Delta E)$ between a probe $P$ and the target $T$ were calculated as:

$$
\Delta E=E_{P, T}-\left[E_{P}+E_{T}\right]
$$

where $E_{P, T}, E_{P}$, and $E_{T}$ are the energies of the probe-target dimer, the probe, and the target, respectively. Interaction energies at optimal geometries indicate the maximum strength of a given $\mathrm{HB}$ pair.

For a subset of 20 donors and 44 acceptors, representative of the strength spectrum considered, we enumerated all pairwise combinations at optimal geometries (880 dimers), generating the panel in Fig.S9. Interaction energies present a systematic and continuous trend, showing that relative strength ranking of a monovalent $\mathrm{HB}$ group is independend of the molecule used to probe it. ${ }^{36-39}$ For this reason, we chose $\Delta E$ values calculated with hydrogen cyanide $(\mathrm{HCN})$ to rank the strengths of all donors (as compound 61) and acceptors (as compound 24). HCN is a linear molecule, sterically unhindered and highly polarized, minimizing the relative contribution of dispersion interactions and steric clashes. Interaction energies calculated in vасиo allow a raw assessment of their competitive strength with respect to water, which was expressly included in the dataset (as compounds $\mathbf{1 5}$ and 75). Overall, strengths of neutral acceptors and donors in the dataset ranges from 0.3 to 1.7 times that of water (Fig.S7 and Fig.S8). Charged groups show a 4- to 10-fold increase with respect to their corresponding neutral moieties.

Donor strength (Fig.S7) correlates with high electronegativity and low oxydation state of the heavy atom to which hydrogen is bound, and is further modulated by the groups attached to it. Sulfur and carbon donors are weaker donors than water (with the notable exception of HCN 61), while nitrogen- and oxygen-containing donors are generally stronger. Acceptor strength (Fig.S8) depends on the availability of electrons to interact with the donor proton, therefore it is modulated by atoms directly bound to the acceptor atom, and the degree in which electrons are shared.

When the same group can act as both acceptor and donor, the respective strengths tend to be negatively correlated. For example, the hydroxyl in phenol is stronger than water when acting as a donor, but weaker when acting as acceptor (compounds 26 and 55, respectively, Fig.S9). The same principle applies to the chemical environment of HB capable groups. Electron-withdrawing substituents decrease acceptor strength, but increase donor strength.

Amino acid ranking. The HB strength calculated for groups contained in amino acid side chains can be used to assess their relative strength. Even though in biological macromolecules their physico-chemical properties will depend on the local environment resulting from folding, these values can provide a rough but insightful gauge of their HB capabilities. According to our calculations, the relative strength of $\mathrm{HB}$ donors is: $\mathrm{Lys}^{+}(\mathbf{3 4}), \mathrm{His}^{+}(\mathbf{3 2}), \mathrm{Arg}^{+}(\mathbf{3 1}) \gg \mathrm{Glu} / \mathrm{Asp}$ (27), Tyr (26) $>$ His (25) $>\operatorname{Trp~(22)~}>$ Gln/Asn (20), backbone amide (18) $>\operatorname{Ser} / \operatorname{Thr}(\mathbf{1 6})>$ water $(\mathbf{1 5})>\operatorname{Arg}(\mathbf{1 3})>$ Lys (6) $>$ Cys (3). For HB acceptors the rank is: $\mathrm{Asp}^{-} / \mathrm{Glu}^{-}$ (101) $\gg \operatorname{Arg}($ 95) $>$ His (92), Lys (91), backbone amide
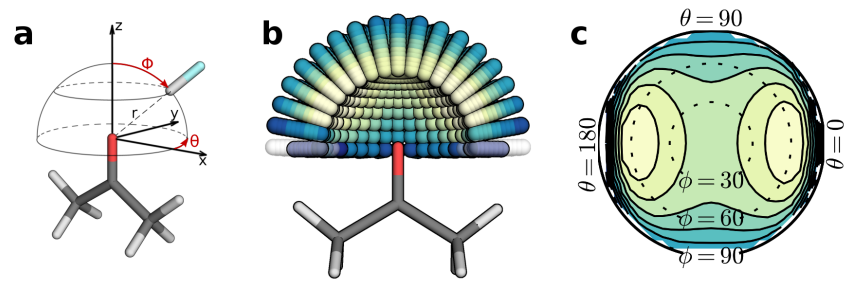

Fig. 1 Methodology to probe and visually represent anisotropy. a Definition of spherical coordinates $(r, \theta, \phi)$ for sampling interaction energies. b Interaction dome. Each sphere corresponds to a position of the probe atom for which an interaction energy was calculated. c Lambert azimuthal equal-area projection (see details in the Methods).

oxygen $(\mathbf{9 0})>$ Asn/Gln $(\mathbf{8 5})>$ water $(\mathbf{7 5})>$ Asp/Glu carbonyl (74) $>$ Ser/Thr (71) $\gg$ Met (56), Tyr (55), Cys (53) > Asp/Glu hydroxyl (43), disulfide (42), Phe (41).

Anisotropy. We studied the anisotropy of 9 donors and 39 acceptors representative of the chemical space and strength spectrum of the full dataset (see Fig. 2 and Supplementary Figs. S4, S5, S10, S11, S12, S13, S14, S15, S16, S17 and S18).

Starting from the optimized dimer coordinates, possible geometries around HB minima were explored for each target atom, mapping variations of $\Delta E$ as a function of the probe position (Fig. 1a). Interaction energies at non-optimal geometries reveal the loss of energy associated with each angular component, i.e. the anisotropy profile. For each target, which can be either a donor or an acceptor, we built a tridimensional map of the interactions, called "dome" (Fig. 1b). Domes were built by moving the probe in a spherical coordinate system $(r, \theta, \phi)$ centered on the target atom (Fig. 1a). To visualize the relationship between interaction energy and angular components, we plotted $\Delta E$ as a function of $\theta$ and $\phi$ using the Lambert azimuthal equal-area projection (Fig. 1c). For each point in the surface defined by $\theta$ and $\phi$ the dome point associated with the optimal distance $r$ (i.e., with the lowest $\Delta E$ ) was chosen.

Anisotropy was quantified by defining the specific directionality index for a given probe as following:

$$
D_{[\text {probe }]}=\frac{1}{N} \sum_{p}^{N} \frac{\Delta E_{o p t}-\Delta E_{p}}{\Delta E_{\text {opt }}}
$$

where $N$ is the total number of accessible dome points (i.e., where interaction energy $\Delta E \leqslant 0), \Delta E_{\text {opt }}$ is the best energy in the dome (i.e., at the optimal geometry), and $\Delta E_{p}$ is the interaction energy with the probe at the point $p$. Very directional HBs have $D_{[p r o b e]}$ values closer to 1 , while isotropic ones (or dimers interacting mostly via dispersion interactions) have values approaching 0 . To quantify the average energy loss at any non-optimal geometry point, we defined the isotropy error $\varepsilon_{[\text {probe }]}$ as the product of $D_{[\text {probe }]}$ with its optimal interaction energy with the probe:

$$
\varepsilon_{[\text {probe }]}=D_{[\text {probe }]} \times \Delta E_{\text {opt }[\text { probe }]}
$$

The anisotropy metrics $D_{[\text {probe }]}$ and $\varepsilon_{[\text {probe }]}$ provide complementary information. While $\varepsilon_{[\text {probe }]}$ is expressed in $\mathrm{kcal} / \mathrm{mol}$, 


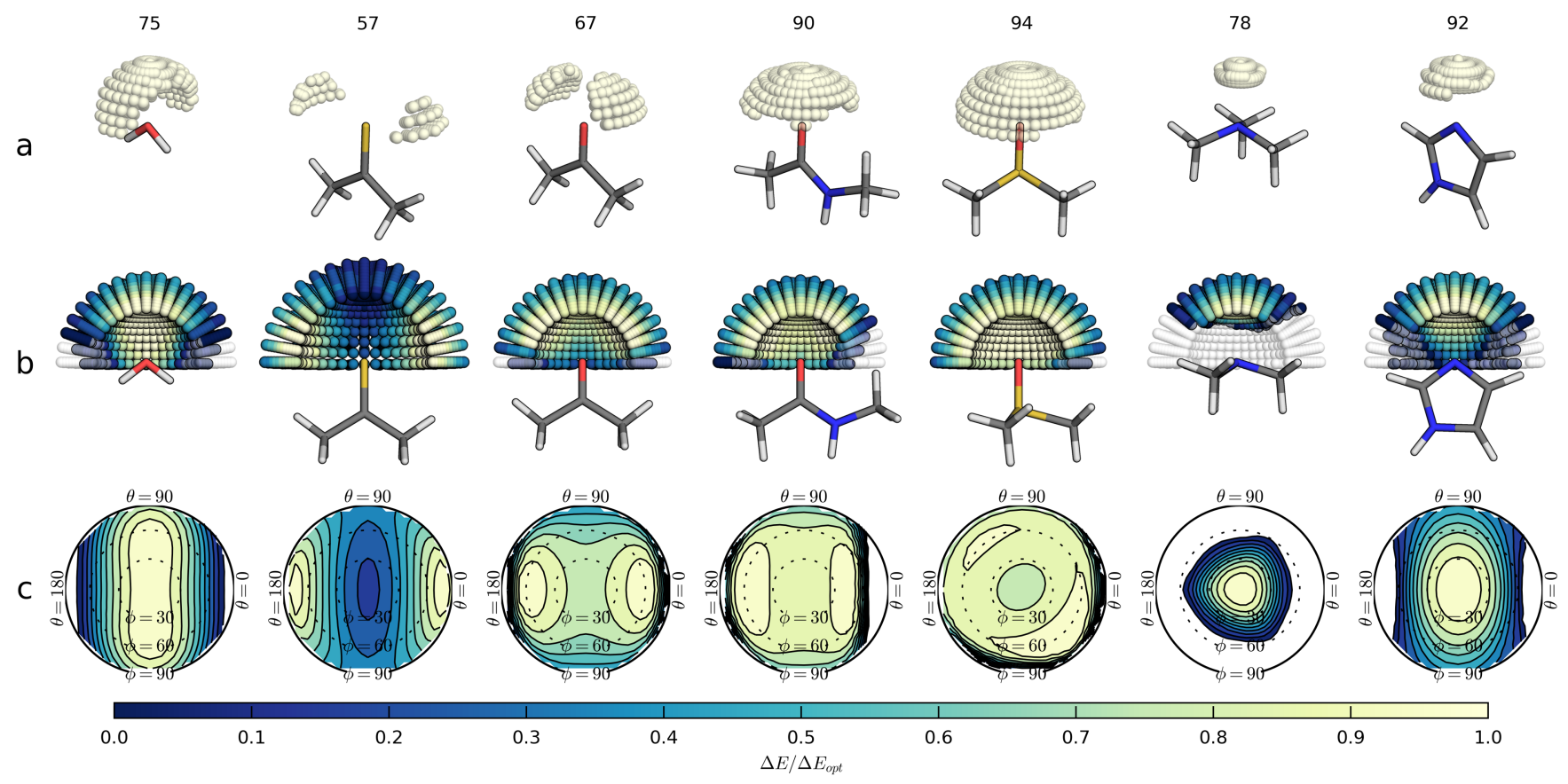

Fig. 2 Domes of a series of acceptor groups (water 75, thioacetone $\mathbf{5 7}, \mathbf{N}$-methyl acetamide $\mathbf{9 0}$, DMSO 94, trimethylamine $\mathbf{7 8}$ and imidazole $\mathbf{9 2}$ ). a Dome points for which $\Delta E$ is $<80 \%$ of $\Delta E_{\text {opt }}$. $\mathbf{b}$ Interaction domes. $\mathbf{c}$ Equal area projections $(\theta, \phi)$ at optimal $r$ distance. Each dome point in a and $\mathbf{b}$ corresponds to a position sampled by the donor probe atom, and is colored according to the normalized $\Delta E / \Delta E_{\text {opt }}$ value. Points and areas with positive $\Delta E$ values (i.e. repulsions) are transparent (a, b) or white (c).

$D_{[\text {probe }]}$ is dimensionless and normalized, making it insensitive to the magnitude of $\Delta E$.

Both metrics are independent from the absolute amount of surface area accessible to the probe. When two target atoms have similar $D_{\text {[probe] }}$ values, they share the same fraction of accessible surface showing anisotropy for a HB probe. Furtheremore, these metrics are insensitive to the derivative of the interaction energy with respect to angular components. For example, methylamine (91), dimethylamine (83) and trimethylamine (78) show progressively smaller accessible surfaces due to the increasing hindrance of the methyl groups, as well as a steeper change in interaction energy with an increase in the $\phi$ angle (Supplementary Fig. S11), but $D_{\text {[probe] }}$ values remain almost identical (Table 1$)$. This happens because the surface associated to $\Delta E_{\text {opt }}$ values is reduced as well, due to the diminished HB capable volume, which preserves the same ratio with respect to the total accessible surface.

The calculated values of $D_{[\text {probe }]}$ were then used to analyze the molecules in our dataset, and characterize their anisotropy. Similarly to what done for the strength analysis, we selected probes with the least hindered geometries, as well as the strongest HB interactions, in order to increase the magnitude of interaction energies and minimize the relative contribution of dispersion interactions. Therefore, HCN (61) was selected to probe donors, and HF (30) to probe acceptors. Donors show rather uniform anisotropies, with $D_{[\mathrm{HCN}]}$ ranging between 0.3 and 0.5 , which depend almost entirely on the $\phi$ angle (i.e., the D-H $\cdots$ A angle), with minor variations induced by the different molecular shapes (Supplementary Figs. S10 and S5). Acceptors, on the other hand, present a much larger spectrum of variations, from nearly isotropic to very directional (Fig. 2). Variations in closely related chemical groups show that the broader molecular environment of the acceptor atom, up to two covalent bonds distance, must be considered (Table 1). For example, replacing one of the methyl groups of acetone $67\left(D_{[H F]}=0.29\right)$ with nitrogen in amides $(\mathbf{8 0}, \mathbf{8 5}, \mathbf{8 7})$ reduces $s p^{2}$ oxygen $D_{[H F]}$ to $0.21-0.24$ (Fig. 2). Secondary amide 90 is the least directional carbonyl (Table 1), and the most tolerant to angular deviations, retaining $80 \%$ of $\Delta E_{\text {opt }}$ at $\phi=0$ while acetone 67 retains less than $75 \%$ (Fig. 2). Variations of $\theta$ (i.e., out-of-plane) are also more tolerated in amides than in acetone, with strengths of $50 \%$ and $30 \%$ of those at optimal geometries, respectively, when both $\theta$ and $\phi$ approach $\pm 90^{\circ}$ (Fig. 2). Such overall increased tolerance is likely responsible of the high propensity of proteins backbone to form stable $\mathrm{HBs}$ that are co-linear with the $\mathrm{C}=\mathrm{O}$ bond such as in $\alpha$-helices ${ }^{5,25}$.

When oxygen is bound directly to sulfur, as in DMSO 94, the partial triple bond characteristics ${ }^{40}$ increases acceptor strength to about $140 \%$ that of water, and further reduce directionality ( $D_{[H F]}=0.19$, Fig. 2 ) due to the lone pair delocalization. Similar effect is observed in sulfate 58, sulfonamide $\mathbf{6 5}$, sulfones $(\mathbf{7 6}, \mathbf{7 7})$ and phosphorous $\mathbf{8 9}$, with directionality ranging between 0.20 and 0.16 (Table 1, Fig. S13), and strength between $74 \%$ to $120 \%$ of that of water. Anisotropy profiles of sulfur and phosphate oxyacids (Supplementary Fig. S13). appear compatible with hydration patterns identified experimentally ${ }^{41,42}$.

Sulfur itself is a weaker acceptor than oxygen but shows the same anisotropy change observed between acetone and amides. From thioacetone $\mathbf{5 7}$ to N-methylthioacetamide 62 (Table 1, Fig. S12), the increase in favorable out-of-plane interactions (i.e., more isotropic interaction) is accompanied by 


\begin{tabular}{|c|c|c|c|c|c|}
\hline & $D_{[H F]}$ & $\Delta E_{\text {opt }(H F)}$ & $\varepsilon_{[H F]}$ & element & hybridization \\
\hline hydrogen fluoride (52) & 0.08 & -5.0 & -0.39 & $\mathrm{~F}$ & $s p^{3}$ \\
\hline fluoromethane $\mathbf{5 0}$ ) & 0.10 & -5.0 & -0.48 & $\mathrm{C}$ & $s p^{3}$ \\
\hline acetate (101) & 0.11 & -26.3 & -2.74 & $\mathrm{O}$ & ionic \\
\hline methanethiolate (100) & 0.13 & -25.2 & -3.25 & $\mathrm{~S}$ & ionic \\
\hline methanesulfonamide (65) & 0.16 & -8.0 & -1.20 & $\mathrm{O}$ & $s p^{2(*)}$ \\
\hline dimethyl disulfone (77) & 0.16 & -8.8 & -1.32 & $\mathrm{O}$ & $s p^{2(*)}$ \\
\hline dimethyl sulfoxide (94) & 0.18 & -12.3 & -2.27 & $\mathrm{O}$ & $s p^{2(*)}$ \\
\hline trimethyl phosphate $(\mathbf{8 9})$ & 0.19 & -10.6 & -1.93 & $\mathrm{O}$ & $s p^{2(*)}$ \\
\hline dimethyl sulfate $\mathbf{( 5 8 )}$ & 0.20 & -7.1 & -1.30 & $\mathrm{O}$ & $s p^{2(*)}$ \\
\hline fluorodimethylborane (49) & 0.20 & -4.6 & -0.90 & $\mathrm{~F}$ & $s p^{3}$ \\
\hline $\mathrm{N}$-methylacetamide $(\mathbf{9 0})$ & 0.21 & -10.6 & -2.15 & $\mathrm{O}$ & $s p^{2}$ \\
\hline acetamide $\mathbf{8 5}$ ) & 0.24 & -10.9 & -2.66 & $\mathrm{O}$ & $s p^{2}$ \\
\hline $\mathrm{N}, \mathrm{N}$-dimethylacetamide $(\mathbf{8 8})$ & 0.25 & -10.2 & -2.60 & $\mathrm{O}$ & $s p^{2}$ \\
\hline methyl acetate (72) & 0.25 & -8.2 & -2.17 & $\mathrm{O}$ & $s p^{2}$ \\
\hline TMAO (97) & 0.25 & -17.2 & -4.28 & $\mathrm{O}$ & $s p^{2} / s p^{3}$ \\
\hline benzamide $(\mathbf{8 0})$ & 0.27 & -9.9 & -2.77 & $\mathrm{O}$ & $s p^{2}$ \\
\hline nitrobenzene (59) & 0.29 & -6.4 & -1.80 & $\mathrm{O}$ & $s p^{2} / s p^{3}$ \\
\hline acetone $(67)$ & 0.29 & -9.0 & -2.56 & $\mathrm{O}$ & $s p^{2}$ \\
\hline hydrogen cyanide $(\mathbf{6 1})$ & 0.29 & -6.5 & -1.89 & $\mathrm{~N}$ & $s p$ \\
\hline water (75) & 0.34 & -8.6 & -2.98 & $\mathrm{O}$ & $s p^{3}$ \\
\hline methyl isocyanide (73) & 0.36 & -8.5 & -3.02 & $\mathrm{C}$ & $s p$ \\
\hline $\mathrm{N}$-methylthioacetamide (62) & 0.36 & -8.3 & -2.95 & $\mathrm{~S}$ & $s p^{2}$ \\
\hline methanol (71) & 0.36 & -8.4 & -3.07 & $\mathrm{O}$ & $s p^{3}$ \\
\hline dimethyl ether $(\mathbf{6 3})$ & 0.41 & -8.1 & -3.29 & $\mathrm{O}$ & $s p^{3}$ \\
\hline phenol (55) & 0.42 & -6.2 & -2.58 & $\mathrm{O}$ & $s p^{3}$ \\
\hline acetic acid (43) & 0.44 & -5.3 & -2.27 & $\mathrm{O}$ & $s p^{3}$ \\
\hline imidazole (92) & 0.45 & -11.6 & -5.20 & $\mathrm{~N}$ & aromatic \\
\hline furan (45) & 0.46 & -4.7 & -2.15 & $\mathrm{O}$ & aromatic \\
\hline dimethyl disulfide (42) & 0.47 & -5.2 & -2.12 & $\mathrm{~S}$ & $s p^{3}$ \\
\hline methyl acetate (46) & 0.48 & -5.7 & -2.66 & $\mathrm{O}$ & $s p^{3}$ \\
\hline dimethyl sulfide (56) & 0.48 & -6.5 & -3.04 & $\mathrm{~S}$ & $s p^{3}$ \\
\hline pyridine (82) & 0.53 & -10.6 & -5.64 & $\mathrm{~N}$ & aromatic \\
\hline methanethiol (53) & 0.50 & -5.7 & -2.82 & $\mathrm{~S}$ & $s p^{3}$ \\
\hline thioacetone $(57)$ & 0.50 & -7.2 & -3.49 & $S$ & $s p^{2}$ \\
\hline $\mathrm{N}$-methylacetonimine (86) & 0.51 & -12.3 & -6.26 & $\mathrm{~N}$ & $s p^{2}$ \\
\hline methanesulfonamide (48) & 0.53 & -7.0 & -3.68 & $\mathrm{~N}$ & $s p^{2} / s p^{3}$ \\
\hline dimethylamine $(\mathbf{8 3})$ & 0.54 & -11.6 & -6.24 & $\mathrm{~N}$ & $s p^{3}$ \\
\hline trimethylamine (78) & 0.55 & -11.0 & -6.04 & $\mathrm{~N}$ & $s p^{3}$ \\
\hline methylamine (91) & 0.55 & -12.0 & -6.60 & $\mathrm{~N}$ & $s p^{3}$ \\
\hline
\end{tabular}

an increase in the magnitude of $\Delta E_{\mathrm{opt}}$.

Overall, nitrogen has a sharper directional character than oxygen as its optimal interaction regions more precisely defined (Figs. 3 and S11). Its relatively limited range of variations across the dataset suggests less dependency on hybridization or molecular environment. Aromatic and $s p^{2}$ nitrogens show minor broadening of the optimal interaction regions, above and below planes of the ring (see imidazole 92 in Fig. 2 and pyridine 82, $N$-methylacetoimine 86 and guanidine 95 in Supplementary Fig. S16). Interestingly, the nitrogen in sulfonamide 48 shows a very high $D_{[H F]}$ value similar to amines $(\mathbf{9 1}, \mathbf{8 3}, \mathbf{7 8})$ despite being a weaker HB acceptor ( $<75 \%$ the strength of amines).

In charged species, such as thiolate $\mathbf{1 0 0}$ and carboxylate $\mathbf{1 0 1}$ (Supplementary Fig. S17), the increased acceptor strength is associated with a sharp $D_{[H F]}$ reduction with respect to their corresponding neutral forms, and independently from the initial anisotropy: from methanethiol $\mathbf{5 3}$ to thiolate $\mathbf{1 0 0}$,
$D_{[H F]}$ drops from 0.5 to 0.13 . from carboxylic acid $\mathbf{7 4}$ to carboxylate 101, $D_{[H F]}$ drops from 0.24 to e 0.11 . Additional acceptor directionality patterns are shown in Supplementary Figs. S14, S15 and S16.

Anisotropy in cooperative HBs. Molecules in the condensed phase tend to be more polarized than in the gas phase ${ }^{43}$ because of the eletrostatic stabilization provided by the adjacent molecules. This cooperative effect plays an important role for HB networks where molecules act as donors and acceptors simultaneously ${ }^{44}$.

To study the impact of cooperativity on the directionality profile of donor and acceptor sites, we considered three molecules capable of forming multiple HBs: water (15 and 75), imidazole (25 and 92) and $N$-methylacetamide (18 and 90). To each target molecule $(T)$ we added a water molecule $(W)$ to one donor or acceptor site (Fig. S18), then we adapted eq.1 to calculate the interaction energy $\Delta E_{\text {wat }}$ with the probe 
$P$ at the free site:

$$
\Delta E_{w a t}=E_{P T W}-\left[E_{P}+E_{T W}\right]-\Delta E_{P W}
$$

where $E_{P T W}, E_{P}$, and $E_{T W}$ are, respectively, the absolute energies of the probe-target-water trimer, the probe alone, and the target-water dimer. $\Delta E_{P T}$ is the interaction energy between the probe and the water, which can be expanded as $E_{P W}-\left[E_{P}+E_{W}\right]$. The polarization induced by the water molecule ${ }^{44}$ affects strengths of both probe-target and target-water interactions. The result is a strength increase for all six sites considered, with $\Delta E_{\text {wat }}$ approximately $3 \%$ to $25 \%$ larger than the corresponding $\Delta E_{\text {opt }}$ of dimers without the added waters (Supplementary Tables S3 and S4). On the other hand, directionalty mapped using the same scheme applied for dimers in the gas phase showed no significant changes, with profiles that were virtually indistinguishable from the non-cooperative ones (Fig. S18). The results suggest that anisotropy on the gas phase is transferable to the condensed phase.

\section{Discussion}

Anisotropy is one of the key features of $\mathrm{HB}^{45,46}$ but its directional preferences are often overlooked in recent literature. While many optimal geometry configurations have been described $^{47-49}$, there is still considerable uncertainty on how strength decays when deviating from these conditions, especially for acceptors. For example, the interaction angle at the acceptor atom is not considered as a criterion for HB identification, while the donor angle is ${ }^{9}$. While it is true that some acceptors are nearly isotropic, others show strong directional preferences.

Retrospective statistical analyses on structural databases are arguably more biased against weak HB interactions, which are either under- ${ }^{46}$ or mis-represented by the influence of stronger forces. This is particularly relevant in the constrained conditions of solid state ${ }^{9,46}$.

The focus of this work was to characterize the HB properties of wide range of chemical groups capable of HB interactions, especially of the interplay between strength and directionality. In order to do that, we designed a method to quantify directionality, which allowed us to compare such properties between different chemical groups. QM calculations were used to characterize variations of $\mathrm{HB}$ interaction energies at high level of detail, while minimizing interferences from non-HB interactions, and free from the packing bias of the condensed phase state.

The initial strength assessment showed a broad and robust trend spanning the whole dataset, which confirmed the additive nature of the interaction in the gas phase and highlighted the transferability properties of single, non-cooperative HBs. That is, while overall strength is the result of respective donor and acceptor strengths, their individual strengths are independent of each other. Calculations for most of the molecules were performed in vacuo, but water itself was part of the dataset to provide a reference value for the assessment of strengths of the other molecules with respect to the biological solvent. Whether or not two solute molecules can form a stable HB in the presence of solvent depends on the relationship between relative strenghts of solute-solute, solutesolvent and solvent-solvent interactions. For that, gas phase interaction energies normalized with respect to water can be used to provide insight on single HBs between solute and solvent, and assess their competitive roles. For a subset of them, we analyzed the effect of cooperative HBs, showing how they affect the interaction strength with respect to energies calculated in vacuo.

Next, uniform geometry sampling was used to build detailed directionality maps probing the volume around HB atoms. To have a consistent metric to quantify anisotropy, we defined a directionality index $D_{[\text {probe }]}$, which makes possible to quantify interactions with a probe to compare and rank different groups.

Results confirmed that all donors present rather similar directional propensity, with minor variations across the set (Supplementary Fig. S5) and in agreement with previous reports ${ }^{35,50,51}$. Acceptors, on the other hand, were found to be anywhere from nearly isotropic to very directional (Fig. 3 ). In very isotropic acceptors, such as DMSO 94 in which $D_{[H F]}$ values are lower, most of the accessible surface can be engaged by a donor hydrogen without significant loss of interaction energy relatively to the optimal interaction geometry. Conversely, highly directional acceptors show larger $D_{[H F]}$ values in the $0.4-0.5$ range, which are close to those of HB donors.

Whereas strength variations can be rationalized basing on properties such as electronegativity, hybridization and acidity, directionality showed to be more complex and difficult to reconcile than previously hypothesized. Clusters in Fig. 3a suggests that the element at an acceptor site is an important factor regulating the overall general strength and directionality characteristics of HB acceptors. Nitrogens are typically strong anisotropic acceptors, occupying the top right corner of the plot. Notably, among acceptors considered here, no other element is simultaneously as strong and anisotropic as nitrogen. Fluorine is simultaneously weak and isotropic (Fig. S16), occupying the opposite corner of the plot with respect to nitrogen. Sulfur is a weak acceptor but displays large anisotropy, occupying the top left corner of the plot. Oxygen displays the widest trend, ranging from weak to strong and from isotropic to directional, but it is neither simultaneously strong and directional like nitrogen, nor weak and isotropic like fluorine. In fact, in oxygen acceptors, strength and $D_{[H F]}$ are anti-correlated such that the stronger the acceptors, the least they manifest directional propensity.

Overall, these results challenge the established consensus according to which strength and directionality are correlated, with the latter depending on the former. Therefore, strong acceptors are expected to be more directional ${ }^{35,45,52-54}$, and weak ones less so ${ }^{35,45,54,55}$. When quantifying directionality of acceptors and donors separately, we found no such correlation with acceptor strength For example, in the same molecular context, sulfur shows more directional character than oxygen, despite the former being a weaker acceptor (Fig. 2). When interacting with HF 30 probe, thioacetone $\mathbf{5 7}$ is $70 \%$ 

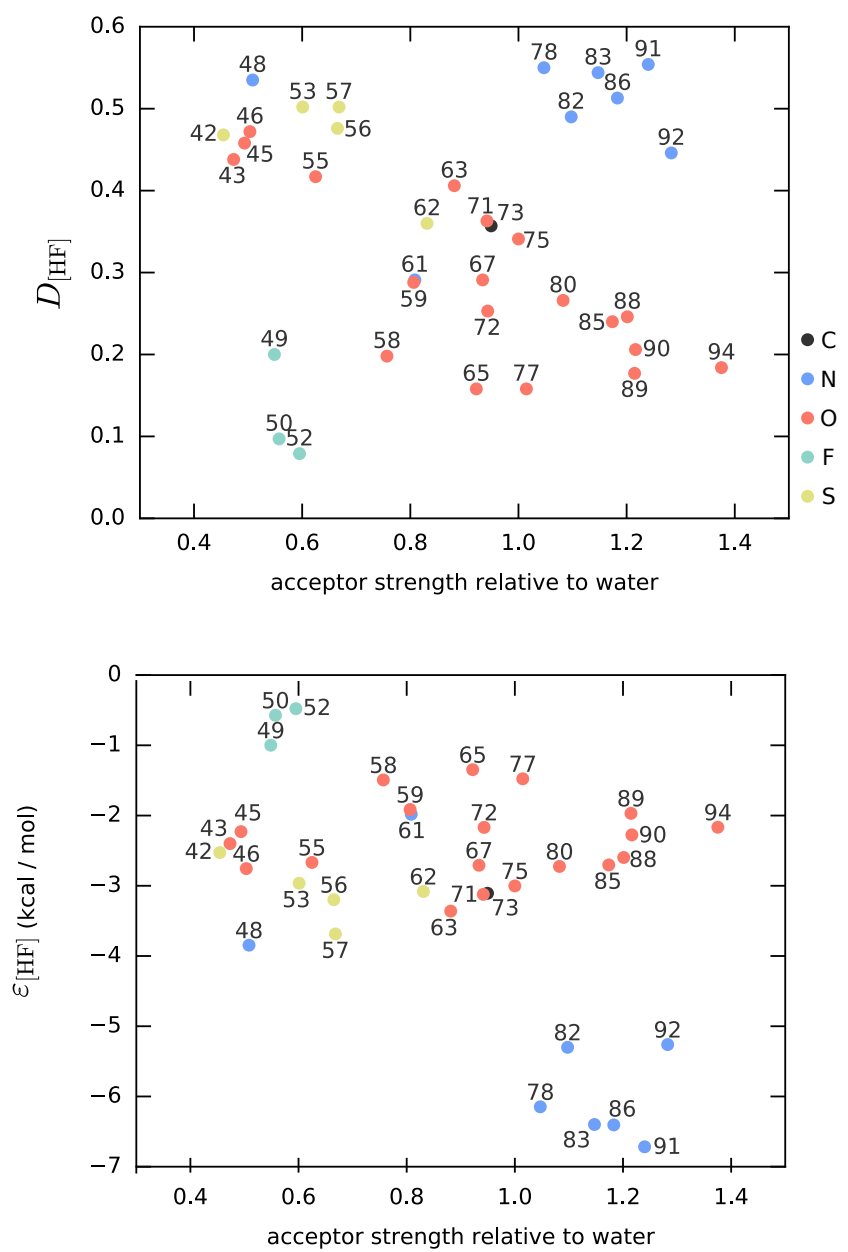

Fig. 3 Directionality and energy errors of molecules in the dataset; points are colored by element type (black carbon; blue nitrogen; red oxygen; cyan fluorine; yellow sulfur) (a) $D_{[H F]}$ values over acceptor strength normalized with respect to water; higher $D_{[H F]}$ values correspond to more directional acceptors; strong and directional nitrogens tend to cluster on the top-right corner, weak and isotropic fluorines on the bottom-left corner, and weak and anisotropic sulfur are on the top-left corner; oxygen atoms are distributed across strengths and directionality $(b)$ quantitative directionality error $\varepsilon_{[H F]}$ versus acceptor strength normalized with respect to water; lower values correspond to higher average energy loss when not establishing optimal HBs

more directional than acetone $67\left(D_{[H F]} 0.50\right.$ and 0.29 , respectively; Table 1) and $20 \%$ weaker $\left(\Delta E_{\text {opt }}-6.95\right.$ and -8.81 $\mathrm{kcal} / \mathrm{mol}$, respectively; Fig. S9).

However, one might argue that high anisotropy of weak acceptors would not translate into effective directional propensity, given the small energies involved. Quantitative isotropy error $\left(\varepsilon_{[H F]}\right)$ can be used to estimate and compare these differences. In absence of external factors, the estimated energy loss at non-optimal geometries would be $0.9 \mathrm{kcal} / \mathrm{mol}$ higher for thioacetone than for acetone $\left(\varepsilon_{[H F]}\right.$ values -3.49 $\mathrm{kcal} / \mathrm{mol}$ and $-2.56 \mathrm{kcal} / \mathrm{mol}$, respectively), revealing the more anisotropic character of the weaker acceptor, even when considering the strenght of the interaction. The lack of correlation between $\varepsilon_{[H F]}$ and acceptor strength is the proeminent observation in Fig. 3b. Notably, acceptor nitrogens are the only element to reach $\varepsilon_{[H F]}$ below $-3.0 \mathrm{kcal} / \mathrm{mol}$, a conse-
Table 2 Variations of directionality values calculated with MP2 for imidazole, acetone, and DMSO using acetylene 12 ( $\left.D_{[\text {acetylene] }}\right)$, water 15 ( $\left.D_{[\text {water }]}\right)$, HCN 24 $\left(D_{[H C N]}\right)$, and HF 20 probes $\left(D_{[H F]}\right)$

\begin{tabular}{rcccc}
\hline & $D_{[\text {acetylene }]}$ & $D_{[\text {water }]}$ & $D_{[H C N]}$ & $D_{[H F]}$ \\
\hline imidazole 92 & 0.32 & 0.35 & 0.34 & 0.43 \\
acetone 67 & 0.18 & 0.23 & 0.19 & 0.27 \\
DMSO 94 & 0.16 & 0.24 & 0.14 & 0.19 \\
\hline
\end{tabular}

Table 3 Interaction energies $\Delta E_{\text {opt }}$ between imidazole, acetone and DMSO and four donors: acetylene 12, water 15, HCN 24 and HF 20. The level of theory is MP2/aug-cc-pVTZ with counterpoise correction.

\begin{tabular}{rcccc}
\hline & $\Delta E_{\text {opt }[\text { acetylene }]}$ & $\Delta E_{\text {opt }[\text { water }]}$ & $\Delta E_{\text {opt }[H C N]}$ & $\Delta E_{\text {opt }[H F]}$ \\
\hline imidazole 92 & -4.3 & -7.2 & -8.1 & -13.4 \\
acetone 67 & -3.4 & -5.9 & -6.1 & -10.0 \\
DMSO 94 & -4.6 & -8.4 & -8.0 & -13.0 \\
\hline
\end{tabular}

quence of being simultaneously strong and anisotropic.

We then studied the influence of the probe strength on the measure of directionality, probing three acceptors (imidazole 92, acetone 67, DMSO 94) using acetylene 12, water 15, HCN 24 and HF 20, and calculated the resulting $D_{[\text {probe] }}$ values (Tables 2 and 3, Fig. 4).

Contrarily to what we found for cooperative HBs, where the presence of an extra $\mathrm{HB}$ with a water molecule affected the overall interaction strength but not directionality, the increase in the probe strength itself induces a systematic directionality increase. The results show that the relative directionality rank between two acceptors is conserved even when sampled with different probes, hinting at an intrinsic directionality property that indeed depends on the sole target characteristics, but gets also modulated by the probe. Notably, a directional acceptor such as imidazole 92 interacting with a very weak HB probe (acetylene 12) maintains more directional character than the more isotropic DMSO 94 interacting with a very strong HB (HF 20). Deviations from the trend were found when probing with a less linear molecule, such as water, due to interactions with flanking atoms (i.e., water hydrogens and acetone and DMSO methyls, Fig. 4a). Another interesting aspect is the effect of the HCN probe on the profiles, suggesting that the $\mathrm{C}-\mathrm{H}$ pair in the nitrile induces similar HB anisotropy to the ethyne in acetylene despite being stronger (Fig. $4 \mathrm{~b}$ and c), possibly due to the association of its strong dipole and medium $\mathrm{HB}$ strenght. ${ }^{56}$

The results require rethinking the way directionality is described, with implications for different aspects in which HBs are involved, and where directionality shapes the energy funnels around acceptors. From a thermodynamic perspective, isotropic acceptors are expected to be potentially more stable in water due to the larger number of microstates with energetically favorable HBs with the solvent, which shift the thermodynamic equilibrium towards the unbound state. Conversely, anisotropic groups (such as acceptor nitrogen) will interact less extensively and in a more ordered fashion with water, hence will pay smaller desolvation penalties upon binding. In fact, introducing a nitrogen acceptor site in a compound is often associated with an increase in potency ${ }^{57}$. Drug design efforts toward improved binders should take into account that the directionality of acceptors can have an impact beyond the direct interaction between ligand and receptor, affecting also 

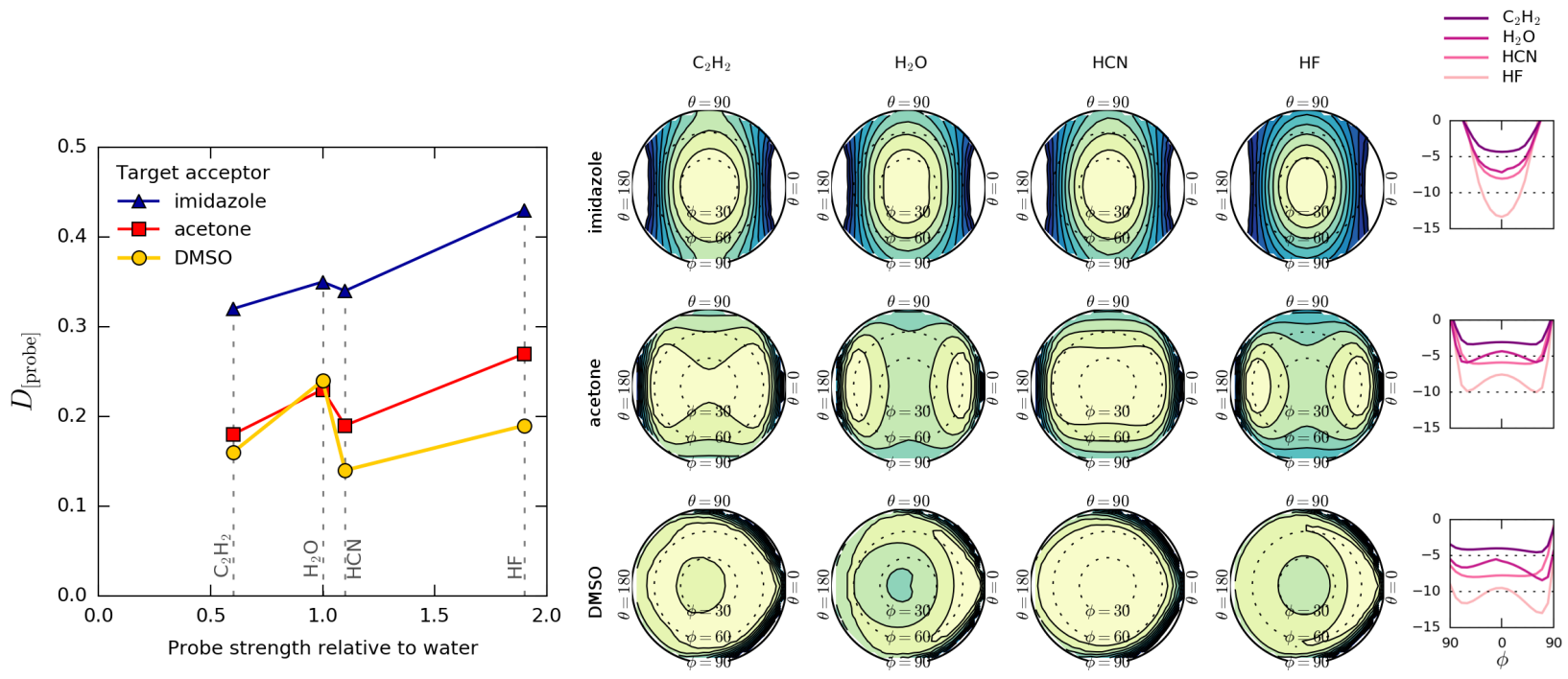

Fig. 4 Directionality profiles and indices $\left(D_{[\text {probe }]}\right)$ of imidazole $\mathbf{9 2}$, acetone $\mathbf{6 7}$, and DMSO 94 using acetylene 12, water 15, HCN 24, and HF 20 probes; a) directionality indices versus water-relative probe strengths; $b$ ) in plane directionality profiles $(\theta=0, \phi=[-90,90]) ; c)$ equal area projections of domes.

their individual interactions with the solvent. In this regard, not all HB groups might be counted equally when defining properties like drug-likeness ${ }^{57}$. From a modeling perspective, the isotropy error $\varepsilon_{[p r o b e]}$ is a useful metric for quantifying the estimate inaccuracy occurring when fully isotropic description (i.e., a spherical model) is used to describe a given acceptor-donor interaction. Generally, more detailed models tend to require more computational resources, therefore having a way to estimate the trade-off between accuracy and speed provides a guidance in the selection of appropriate approximations..$^{50}$

Far from being comprehensive, our dataset represents a compendium of the spectrum of HBs in the context of structural biology and supramolecular chemistry. We must note that in the condensed phase, polarization effects and networks of cooperative HBs modulate the strengths of HB capable molecules, therefore limiting the predictive power of our in vacuo calculations. Nevertheless, the chemical groups considered here provide an overview on how different donors and acceptors compare with each other, and with respect to water. This is particularly useful for chemical biology, protein engineering, drug design, and all cases in which solvation effects temper reversible interactions.

Finally, we believe that the geometric scheme described here, as well as the quantification of the directionality, have a more general interest. The possibility of performing such quantifications and comparisons in a consistent framework allowed us to identify a number of novel patterns. The method is suitable for generating high quality parameters for molecular mechanics force fields used in molecular dynamics and docking simulations, and can be easily adapted and extended to study other anisotropic interactions.

\section{Methods}

Dataset. Donors (1-34, Fig. S1) and acceptors (35-101, Fig. S2) were selected to provide a representative and diverse sample of classical ${ }^{9,58,59}$ and non-conventional ${ }^{55,59} \mathrm{HB}$ groups, with emphasis on those common in biological and drug design contexts. With the exception of the cooperativ- ity analysis, only single-site HBs have been considered to facilitate strength comparisons and directionality analysis, hence excluding multi-furcated interactions. In molecules capable of multiple HBs, each site was considered separately, e.g.: water is compound $\mathbf{1 5}$ as donor, and compound $\mathbf{7 5}$ as acceptor.

Dimer and trimer geometries and strength. All calculations were performed using Gaussian $09^{60}$. Ideal acceptor-donor geometries were constructed and minimized with the B3LYP density functional ${ }^{61-64}$ and the ccpVDZ basis set ${ }^{65}$ and counterpoise correction ${ }^{66,67}$. Calculations were performed in vacuo, while water was considered part of the dataset and used to provide a reference value for the other molecules considered. Optimized structures were tested for absence of imaginary frequencies, and visually inspected to exclude interactions other than HBs, such as the formation of Lewis adducts or geometries that are clearly far from an ideal HB geometry. (i.e., interactions involving atoms other than the target/probe pairs, such as DMSO (92) methyls with HF (30) fluorine). Dimers and trimers that had optimized to transition states or to multi-furcated interactions were re-optimized from different starting geometries until issues were resolved. Electronic interaction energy $\Delta E$ of the minimized dimers calculated with eq. 1 was used to assess HB strength. The absolute energy of the isolated (unbound) monomers corresponds to their optimized geometries in gas-phase (as opposed to their optimized geometries in the dimer). The impact of the choice of the density functional is discussed in the Supplementary Information.

Directionality. The reference frame system defined the position of the probe (Fig. 1a) was defined as following: $a$ ) the reference center is set at the target atom, such that spherical coordinate $r$ represents its distance with the probe; $b$ ) the $z$-axis is aligned along the mean of the bond vectors between neighbor atoms of the target and the target atom itself; $c$ ) the $x$ axis is defined as the vector orthogonal to the $z$ axis and oriented such that the bond to the bulkiest group in the molecule lies in positive $x$ direction. Directional preferences of donors and acceptors were derived by sampling angular components $(\phi, \theta)$ and distance $(r)$, keeping the probe aligned to the $r$ vector. Angular components were sampled at intervals of 10 degrees, from 0 to 90 for $\phi$ (10 intervals), and 0 to 360 for $\theta$ (37 intervals). Distance $r$ was sampled at intervals of $0.1 \AA$ from optimized dimer geometry up to $1 \AA$ away (11 intervals), to accommodate for possible repulsion when leaving the $\mathrm{HB}$ well. Whenever possible, molecular simmetry was used to reduce the number of dome points to calculate. For most molecules, QM calculations were performed for $\theta$ values in the range $0-180$, then equal area projections where generated by applying simmetry transformations. Exceptions were dimethyl sulfoxide 94 and trimethylphosphate 89, for which QM calculations were performed covering the entire 0-360 range. During the geometry scanning, internal geometries (i.e.: bond lengths and angles) of target and probe were kept fixed, in order to preserve the intended $(r, \phi, \theta)$ values. The valida- 
tion of the level of theory to measure directionality, and the use of internal geometry restraints are discussed in Supplementary Methods.

Lambert azimuthal equal-area projection. The Lambert azimuthal equal-area projection was used to represent interaction energies as a function of the angular components. This projection has been used in prior work ${ }^{14}$. For each $\theta$ and $\phi$ pair, the lowest $\Delta E$ energy at the optimal $r$ distance was represented in the projection. To generate the equal area projections, the position of probe atoms was converted from cartesian coordinates $\hat{r}=(x, y, z)$ into projection coordinates $(X, Y)$ :

$$
\begin{aligned}
& X=\frac{x}{|\hat{r}|} \sqrt{\frac{2}{1+\frac{z}{\mid \hat{r}} \mid}} \\
& Y=\frac{y}{|\hat{r}|} \sqrt{\frac{2}{1+\frac{z}{|\hat{r}|}}}
\end{aligned}
$$

$\Delta E$ is then represented as a function of projection coordinates $(X, Y)$. Values used for the graphical representation of equal-area projections were obtained with bilinear interpolation of $\Delta E$ values in $(X, Y)$ coordinates. $\Delta E$ values interpolated from eequal-area projection coordinates were used to calculate directionality indices $D_{i}$, to obtain a uniform sampling of the exposed target surface and compensate for variations in $r$ due to different equilibrium distances, and the angular density variations in dome points.

\section{ACKNOWLEDGEMENTS}

The authors thank David S. Goodsell for insightful discussions, and Lisa Dong and J.C. Ducom at the TSRI HPC core for computational support. This work was supported by the National Institutes of Health (GM069832). This is manuscript number 29667 of the Scripps Research Institute. The authors declare that they have no competing financial interests.

\section{Bibliography}

1. Bissantz, C., Kuhn, B. \& Stahl, M. A medicinal chemist's guide to molecular interactions. Journal of Medicinal Chemistry 53, 5061-5084 (2010).

2. Snyder, P. W., Lockett, M. R., Moustakas, D. T. \& Whitesides, G. M. Is it the shape of the cavity, or the shape of the water in the cavity? The European Physical Journal Special Topics 223, 853-891 (2013).

3. Smith, J. D. Energetics of hydrogen bond network rearrangements in liquid water. Science 306, 851-853 (2004)

4. Brini, E. et al. How water's properties are encoded in its molecular structure and energies. Chemical Reviews 117, 12385-12414 (2017).

5. Pauling, L., Corey, R. B. \& Branson, H. R. The structure of proteins: two hydrogen-bonded helical configurations of the polypeptide chain. Proceedings of the National Academy of Sciences 37, 205-211 (1951).

6. Shan, S.-o. \& Herschlag, D. Energetic effects of multiple hydrogen bonds. implications for enzymatic catalysis. Journal of the American Chemical Society 118, 5515-5518 (1996).

7. Alegre-Requena, J. V., Marqués-López, E., Herrera, R. P. \& Díaz, D. D. Metal-organic frameworks (mofs) bring new life to hydrogen-bonding organocatalysts in confined spaces. CrystEngComm 18, 3985-3995 (2016).

8. Steiner, T. \& Desiraju, G. R. Distinction between the weak hydrogen bond and the van der Waals interaction. Chemical Communications 891-892 (1998).

9. Arunan, E. et al. Definition of the hydrogen bond (IUPAC Recommendations 2011). Pure and applied chemistry 83, 1637-1641 (2011).

10. Groom, C. R., Bruno, I. J., Lightfoot, M. P. \& Ward, S. C. The cambridge structural database. Acta Crystallographica Section B Structural Science, Crystal Engineering and Materials 72 , 171-179 (2016).

11. Berman, H. M. The Protein Data Bank. Nucleic Acids Research 28, 235-242 (2000).

12. Desiraju, G. R. lupac definition of the hydrogen bond. terminology and nomenclature. Foundations of Crystallography 70, C308 (2017)

13. Morozov, A. V., Kortemme, T., Tsemekhman, K. \& Baker, D. Close agreement between the orientation dependence of hydrogen bonds observed in protein structures and quantum mechanical calculations. Proceedings of the National Academy of Sciences 101, 69466951 (2004).

14. O'Meara, M. J. et al. Combined covalent-electrostatic model of hydrogen bonding improves structure prediction with rosetta. Journal of chemical theory and computation 11, 609-622 (2015)

15. Saha, B. K., Saha, A., Sharada, D. \& Rather, S. A. F or O, which one is the better hydrogen bond (is it?) acceptor in $\mathrm{C}-\mathrm{H} \cdots \mathrm{X}-\mathrm{C}(\mathrm{X}-=\mathrm{F}-, \mathrm{O}=)$ interactions? Crystal Growth \& Design (2017)

16. Zhou, P., Tian, F., Lv, F. \& Shang, Z. Geometric characteristics of hydrogen bonds involving sulfur atoms in proteins. Proteins: Structure, Function, and Bioinformatics 76, 151-163 (2009)

17. Brammer, L., Bruton, E. A. \& Sherwood, P. Understanding the behavior of halogens as hydrogen bond acceptors. Crystal Growth \& Design 1, 277-290 (2001).

18. Allen, F. H., Bird, C. M., Rowland, R. S. \& Raithby, P. R. Resonance-induced hydrogen bonding at sulfur acceptors in $\mathrm{R}_{1} \mathrm{R}_{2} \mathrm{C}=\mathrm{S}$ and $\mathrm{R}_{1} \mathrm{CS}_{2}{ }^{-}$systems. Acta Crystallographica Section B Structural Science 53, 680-695 (1997).
19. Allen, F., Bird, C., Rowland, R. \& Raithby, P. Hydrogen-bond acceptor and donor properties of divalent sulfur (YSZ and RSH). Acta Crystallographica Section B: Structural Science $\mathbf{5 3}$, 696-701 (1997)

20. Wood, P. A., Pidcock, E. \& Allen, F. H. Interaction geometries and energies of hydrogen bonds to $\mathrm{C}=\mathrm{O}$ and $\mathrm{C}=\mathrm{S}$ acceptors: a comparative study. Acta Crystallographica Section $B$ Structural Science 64, 491-496 (2008).

21. Bruno, I. J. et al. Isostar: A library of information about nonbonded interactions. Journal of Computer-Aided Molecular Design 11, 525-537 (1997).

22. Steiner, T. The hydrogen bond in the solid state. Angewandte Chemie International Edition 41, 48-76 (2002).

23. Zhou, P., Zou, J., Tian, F. \& Shang, Z. Fluorine bonding - how does it work in protein-ligand interactions? Journal of Chemical Information and Modeling 49, 2344-2355 (2009).

24. Voth, A. R., Khuu, P., Oishi, K. \& Ho, P. S. Halogen bonds as orthogonal molecular interactions to hydrogen bonds. Nature Chemistry 1, 74-79 (2009).

25. Nittinger, E. et al. Large-scale analysis of hydrogen bond interaction patterns in proteinligand interfaces. Journal of Medicinal Chemistry 60, 4245-4257 (2017).

26. Allen, F. H., Harris, S. E. \& Taylor, R. Comparison of conformer distributions in the crystalline state with conformational energies calculated by ab initio techniques. Journal of ComputerAided Molecular Design 10, 247-254 (1996).

27. Hendrickson, W. A. Stereochemically restrained refinement of macromolecular structures. In Methods in enzymology, vol. 115, 252-270 (1985).

28. Wendler, K., Thar, J., Zahn, S. \& Kirchner, B. Estimating the hydrogen bond energy. The Journal of Physical Chemistry A 114, 9529-9536 (2010).

29. Kirchner, B. \& Reiher, M. The secret of dimethyl sulfoxide- water mixtures. a quantum chemical study of $1 \mathrm{dmso}-\mathrm{n}$ water clusters. Journal of the American Chemical Society 124 , 6206-6215 (2002)

30. Li, X.-Z., Walker, B. \& Michaelides, A. Quantum nature of the hydrogen bond. Proceedings of the National Academy of Sciences 108, 6369-6373 (2011).

31. Lehmann, S., Spickermann, C. \& Kirchner, B. Quantum cluster equilibrium theory applied in hydrogen bond number studies of water. 1. assessment of the quantum cluster equilibrium model for liquid water. Journal of chemical theory and computation 5, 1640-1649 (2009).

32. Green, A. J. \& Popelier, P. L. Theoretical prediction of hydrogen-bond basicity $\mathrm{p} \mathrm{k} \mathrm{bhx} \mathrm{using}$ quantum chemical topology descriptors. Journal of chemical information and modeling $\mathbf{5 4}$, 553-561 (2014).

33. Dannenberg, J., Haskamp, L. \& Masunov, A. Are hydrogen bonds covalent or electrostatic? a molecular orbital comparison of molecules in electric fields and $\mathrm{h}$-bonding environments. The Journal of Physical Chemistry A 103, 7083-7086 (1999).

34. Umeyama, H. \& Morokuma, K. The origin of hydrogen bonding. an energy decomposition study. Journal of the American Chemical Society 99, 1316-1332 (1977).

35. Shahi, A. \& Arunan, E. Why are hydrogen bonds directional? Journal of Chemical Sciences 128, 1571-1577 (2016).

36. Abraham, M. H., Grellier, P. L., Prior, D. V., Morris, J. J. \& Taylor, P. J. Hydrogen bonding Part 10. a scale of solute hydrogen-bond basicity using log $k$ values for complexation in tetrachloromethane. Journal of the Chemical Society, Perkin Transactions 2521 (1990).

37. Laurence, C., Brameld, K. A., Graton, J., Le Questel, J.-Y. \& Renault, E. The pk database: Toward a better understanding of hydrogen-bond basicity for medicinal chemists. Journal of Medicinal Chemistry 52, 4073-4086 (2009).

38. Klamt, A., Reinisch, J., Eckert, F., Hellweg, A. \& Diedenhofen, M. Polarization charge densities provide a predictive quantification of hydrogen bond energies. Phys. Chem. Chem. Phys. 14, 955-963 (2012)

39. Zheng, S. et al. Proposed hydrogen-bonding index of donor or acceptor reflecting its intrinsic contribution to hydrogen-bonding strength. Journal of Chemical Information and Modeling (2017).

40. Kutzelnigg, W. Chemical bonding in higher main group elements. Angewandte Chemie International Edition 23, 272-295 (1984)

41. Śmiechowski, M., Gojło, E. \& Stangret, J. Systematic study of hydration patterns of phosphoric (V) acid and its mono-, di-, and tripotassium salts in aqueous solution. The Journal of Physical Chemistry B 113, 7650-7661 (2009).

42. Ruben, E. A., Chapman, M. S. \& Evanseck, J. D. Hydrogen bonding mediated by key orbital interactions determines hydration enthalpy differences of phosphate water clusters. The Journal of Physical Chemistry A 111, 10804-10814 (2007)

43. Bayly, C. I., Cieplak, P., Cornell, W. \& Kollman, P. A. A well-behaved electrostatic potential based method using charge restraints for deriving atomic charges: the resp model. The Journal of Physical Chemistry 97, 10269-10280 (1993).

44. Nochebuena, J., Cuautli, C. \& Ireta, J. Origin of cooperativity in hydrogen bonding. Physical Chemistry Chemical Physics 19, 15256-15263 (2017)

45. Pimentel, G. C. \& McClellan, A. L. The hydrogen bond (Freeman, 1960).

46. Steiner, T. \& Desiraju, G. R. Distinction between the weak hydrogen bond and the van der Waals interaction. Chemical Communications 891-892 (1998).

47. Taylor, R., Kennard, O. \& Versichel, W. Geometry of the imino-carbonyl (N-H ... O:C) hydrogen bond. 1. Ione-pair directionality. Journal of the American Chemical Society 105, 5761-5766 (1983)

48. Desiraju, G. R. $\mathrm{C}-\mathrm{H} \cdots \mathrm{O}$ and other weak hydrogen bonds. from crystal engineering to virtual screening. Chemical Communications 2995-3001 (2005)

49. Wood, P. A., Allen, F. H. \& Pidcock, E. Hydrogen-bond directionality at the donor $\mathrm{H}$ atomanalysis of interaction energies and database statistics. CrystEngComm 11, 1563-1571 (2009).

50. Lu, Z., Zhou, N., Wu, Q. \& Zhang, Y. Directional dependence of hydrogen bonds: A densitybased energy decomposition analysis and its implications on force field development. Journal of chemical theory and computation 7, 4038-4049 (2011).

51. Ireta, J., Neugebauer, J. \& Scheffler, M. On the accuracy of dft for describing hydrogen bonds: Dependence on the bond directionality. The Journal of Physical Chemistry A 108, 5692-5698 (2004)

52. Kollman, P. A. Theory of hydrogen bond directionality. Journal of the American Chemical Society 94, 1837-1842 (1972)

53. Jeffrey, G. A. \& Saenger, W. Hydrogen bonding in biological structures (Springer Science \& 
Business Media, 1991).

54. Jeffrey, G. A. \& Jeffrey, G. A. An introduction to hydrogen bonding, vol. 32 (Oxford university press New York, 1997).

55. Desiraju, G. R. \& Steiner, T. The weak hydrogen bond: in structural chemistry and biology vol. 9 (International Union of Crystalography, Monographs on Crystallography, 2001).

56. Jucks, K. \& Miller, R. Infrared spectroscopy of the hydrogen cyanide dimer. The Journal of chemical physics 88, 6059-6067 (1988).

57. "Pennington, L. D. \& Moustakas, D. T. . The Necessary Nitrogen Atom: A Versatile HighImpact Design Element for Multiparameter Optimization. J. Med. Chem. 60, 3552-3579 (2017).

58. Arunan, E. et al. Defining the hydrogen bond: An account (iupac technical report). Pure and Applied Chemistry 83, 1619-1636 (2011).

59. Alkorta, I. \& Elguero, J. Non-conventional hydrogen bonds. Chemical Society Reviews $\mathbf{2 7}$ 163-170 (1998)

60. Frisch, M. J. et al. Gaussian 09 Revision A.02.

61. Becke, A. D. Density-functional thermochemistry. III. the role of exact exchange. The Journal of Chemical Physics 98, 5648-5652 (1993).

62. Lee, C., Yang, W. \& Parr, R. G. Development of the Colle-Salvetti correlation-energy formula into a functional of the electron density. Physical Review B 37, 785-789 (1988).

63. Vosko, S. H., Wilk, L. \& Nusair, M. Accurate spin-dependent electron liquid correlation energies for local spin density calculations: a critical analysis. Canadian Journal of Physics 58, 1200-1211 (1980).

64. Stephens, P. J., Devlin, F. J., Chabalowski, C. F. \& Frisch, M. J. Ab initio calculation of vibrational absorption and circular dichroism spectra using density functional force fields. The Journal of Physical Chemistry 98, 11623-11627 (1994).

65. Dunning, T. H. Gaussian basis sets for use in correlated molecular calculations. i. the atoms boron through neon and hydrogen. The Journal of Chemical Physics 90, 1007-1023 (1989).

66. Boys, S. \& Bernardi, F. The calculation of small molecular interactions by the differences of separate total energies. some procedures with reduced errors. Molecular Physics 19, 553-566 (1970).

67. Simon, S., Duran, M. \& Dannenberg, J. J. How does basis set superposition error change the potential surfaces for hydrogen-bonded dimers? The Journal of Chemical Physics 105, 11024-11031 (1996).

68. Morozov, A. V. \& Kortemme, T. Potential functions for hydrogen bonds in protein structure prediction and design. Advances in Protein Chemistry 1-38 (2005).

69. Řezáč, J., Riley, K. E. \& Hobza, P. Extensions of the S66 data set: More accurate interaction energies and angular-displaced nonequilibrium geometries. Journal of Chemical Theory and Computation 7, 3466-3470 (2011).

70. Llamas-Saiz, A. L., Foces-Foces, C., Mo, O., Yanez, M. \& Elguero, J. Nature of the hydrogen bond: crystallographic versus theoretical description of the $\mathrm{OH} \cdots \mathrm{N}\left(s p^{2}\right)$ hydrogen bond. Acta Crystallographica Section B: Structural Science 48, 700-713 (1992).

71. Platts, J. A., Howard, S. T. \& Bracke, B. R. F. Directionality of hydrogen bonds to sulfur and oxygen. Journal of the American Chemical Society 118, 2726-2733 (1996).

72. Allen, F. H., Baalham, C. A., Lommerse, J. P. M., Raithby, P. R. \& Sparr, E. Hydrogenbond acceptor properties of nitro-o atoms: A combined crystallographic database andab initiomolecular orbital study. Acta Crystallographica Section B Structural Science 53, 10171024 (1997).

73. Nanda, V. \& Schmiedekamp, A. Are aromatic carbon donor hydrogen bonds linear in proteins? Proteins: Structure, Function, and Bioinformatics 70, 489-497 (2007).

74. Wood, P. A., Allen, F. H. \& Pidcock, E. Hydrogen-bond directionality at the donor h atomanalysis of interaction energies and database statistics. CrystEngComm 11, 1563 (2009).

75. Choi, H., Kang, H. \& Park, H. New angle-dependent potential energy function for backbonebackbone hydrogen bond in protein-protein interactions. Journal of Computational Chemistry NA-NA (2009).

76. Choi, H., Kang, H. \& Park, H. Extended morse function model for angle-dependent hydrogen bond in protein-protein interactions. The Journal of Physical Chemistry B 114, 2980-2987 (2010).

77. Lu, Z., Zhou, N., Wu, Q. \& Zhang, Y. Directional dependence of hydrogen bonds: A densitybased energy decomposition analysis and its implications on force field development. Journal of Chemical Theory and Computation 7, 4038-4049 (2011).

78. Tafipolsky, M. \& Ansorg, K. Toward a physically motivated force field: Hydrogen bond directionality from a symmetry-adapted perturbation theory perspective. Journal of Chemical Theory and Computation 12, 1267-1279 (2016).

79. Mondal, A. \& Datta, S. Quantum mechanical electronic structure calculation reveals orientation dependence of hydrogen bond energy in proteins. Proteins: Structure, Function, and Bioinformatics 85, 1046-1055 (2017).

80. Rao, L., Ke, H., Fu, G., Xu, X. \& Yan, Y. Performance of several density functional theory methods on describing hydrogen-bond interactions. Journal of Chemical Theory and Computation 5, 86-96 (2009).

81. Koné, M., Illien, B., Graton, J. \& Laurence, C. B3LYP and MP2 calculations of the enthalpies of hydrogen-bonded complexes of methanol with neutral bases and anions: Comparison with experimental data. The Journal of Physical Chemistry A 109, 11907-11913 (2005).

82. El Kerdawy, A., Tautermann, C. S., Clark, T. \& Fox, T. Economical and accurate protocol for calculating hydrogen-bond-acceptor strengths. Journal of Chemical Information and Modeling 53, 3262-3272 (2013).

83. Hao, M.-H. Theoretical calculation of hydrogen-bonding strength for drug molecules. Journal of Chemical Theory and Computation 2, 863-872 (2006).

84. Graton, J., Le Questel, J.-Y., Maxwell, P. \& Popelier, P. Hydrogen-bond accepting properties of new heteroaromatic ring chemical motifs: A theoretical study. Journal of chemical information and modeling 56, 322-334 (2016).

85. Kim, K. \& Jordan, K. Comparison of density functional and mp2 calculations on the water monomer and dimer. The Journal of Physical Chemistry 98, 10089-10094 (1994).

86. Murashov, V. \& Leszczynski, J. A comparison of the b3lyp and mp2 methods in the calculation of phosphate complexes. Journal of Molecular Structure: THEOCHEM 529, 1-14 (2000).

87. Goerigk, L. \& Grimme, S. A thorough benchmark of density functional methods for general main group thermochemistry, kinetics, and noncovalent interactions. Physical Chemistry Chemical Physics 13, 6670-6688 (2011).

88. Rezac, J., Huang, Y., Hobza, P. \& Beran, G. J. Benchmark calculations of three-body intermolecular interactions and the performance of low-cost electronic structure methods. Journal of chemical theory and computation 11, 3065-3079 (2015).

89. Medders, G. R., Babin, V. \& Paesani, F. A critical assessment of two-body and three-body interactions in water. Journal of chemical theory and computation 9, 1103-1114 (2013). 


\section{Supplementary Information}

Previous QM works. Supplementary Table S1 summarizes previous QM studies published to date, describing HB dimer interactions at non-optimal geometries, with the aim of characterizing variation in $\Delta E$ with angular components. Most of the previous studies considered only one angular component. Others addressed multiple angular components separately (Morozov et al. ${ }^{13,68}$ and Řezáč et al. ${ }^{69}$ ). We could find only a single study in which two angular components were addressed simultaneously for the same target site (Lamas et al. ${ }^{70}$ ).

Supplementary Table S1 Previous QM studies of HB directionality which considered interaction energies at non-optimal geometries (ranked by publication year)

\begin{tabular}{|c|c|c|c|c|c|}
\hline Ref & Year & Acceptors & Donors & Level of theory & Geometric parameters $^{a}$ \\
\hline Lamas et al. ${ }^{70}$ & 1992 & $\mathrm{~N}\left(s p^{2}\right)$ & - & $\mathrm{HF} / 3-21 \mathrm{G}$ & $f(\theta, \phi)$ \\
\hline Platts et al. ${ }^{71}$ & 1996 & $\mathrm{O}\left(s p^{2}, s p^{3}\right) \mathrm{S}\left(s p^{2}, s p^{3}\right)$ & - & multipole electrostatics ${ }^{b}$ & $f(\phi)$ \\
\hline Allen et al. ${ }^{72}$ & 1997 & $\mathrm{NO}_{2}$ & - & $\mathrm{IMPT} / 6-31 \mathrm{G}^{* *}$ & $f(\phi)$ \\
\hline Morozov et al. ${ }^{13,68}$ & 2004 & formamide & formamide & PBE96/aug-cc-pVDZ & $f(\theta), f(\phi)$ \\
\hline Nanda et al. ${ }^{73}$ & 2007 & - & $\mathrm{CH}$ (aromatic) & B3LYP/6-11++G** & $f(\phi)$ \\
\hline Wood et al. ${ }^{20}$ & 2008 & $\mathrm{O}\left(s p^{2}\right) \mathrm{S}\left(s p^{2}\right)$ & - & IMPT/6-31G $* *$ & $f(\phi)$ \\
\hline Wood et al. ${ }^{74}$ & 2009 & - & $\mathrm{OH}\left(s p^{3}\right) \mathrm{CH}\left(s p, s p^{2}\right)$ & IMPT/6-31G** & $f(\phi)$ \\
\hline Choi et al. ${ }^{75}$ & 2009 & $\mathrm{~N}$-methylacetamide & $\mathrm{N}$-methylacetamide & B3LYP/6-311G** & $f(\phi)$ \\
\hline Choi et al. ${ }^{76}$ & 2010 & $\mathrm{O}\left(s p^{2}\right)$ & $\mathrm{NH}\left(s p^{2}\right) \mathrm{OH}\left(s p^{3}\right)$ & B3LYP/6-311G** & $f(\phi)$ \\
\hline Řezáč et al. ${ }^{69}$ & 2011 & 10 & 10 & $\operatorname{CCSD}(\mathrm{t}) / \mathrm{CBS}$ & $f(\phi), f(\theta)$ \\
\hline Lu et al. 77 & 2011 & $\mathrm{O}\left(s p^{2}, s p^{3}\right) \mathrm{N}\left(s p, s p^{2}, s p^{3}\right) \mathrm{S}\left(s p^{2}, s p^{3}\right)$ & - & B3LYP-D3/aug-cc-pVDZ & $f(\phi)$ \\
\hline Tafipolsky et al. ${ }^{78}$ & 2016 & water & - & DFT-SAPT/aug-cc-pVQZ & $f(\phi)$ \\
\hline Mondal et al. ${ }^{79}$ & 2017 & $\mathrm{O}\left(s p^{2}\right)$ & - & M06-2X/6-311+G* & $f(\phi)$ \\
\hline
\end{tabular}

$\theta$ and $\phi$ values as depicted in Fig. 1

${ }^{\mathrm{b}}$ multipoles were derived from MP2 level of theory.

Dataset molecules. The molecules we selected for our dataset are depicted in Supplementary Figs. S1 (acceptors) and S2 (donors). The dataset includes conventional HB groups commonly found in drugs and biological molecules (e.g., hydroxyl, carbonyl, amines, etc.) as well as some inorganic ones (hydrogen fluoride and chloride, fluoroborane, etc.), spanning a wide range of strengths. Only single-site HBs have been considered to facilitate strength comparisons and directionality analysis, thus excluding multi-furcated interactions. Molecules capable of multiple HBs were considered as separate entries in the dataset, e.g.: water is compound $\mathbf{1 5}$ as donor, and compound $\mathbf{7 5}$ as acceptor, and each HB site was considered separately.

Level of theory validation. Given the large number of independent computations to be performed, we employed the B3LYP density functional with the cc-pVDZ basis set. B3LYP is known to systematically underestimate absolute interaction energies ${ }^{80}$, but numerous previously reported works show that it provides accurate ranking of relative HB strengths ${ }^{81-86}$, which is adequate for the scope of this work. As a validation, we performed multiple tests using MP2/aug-cc-pVTZ on a small sample of compounds (using the counterpoise correction), and compared the results obtained with the two levels of theory to assess the error in both HB strengths and anisotropy profiles.

Accuracy of $H B$ strengths. We compared the values of $\Delta E_{\text {opt }}$ of dimer geometries fully minimized with B3LYP/cc-pVDZ and MP2/aug-cc-pVTZ levels of theory. A small subset of 33 dimers was used for this comparison: dimethylamine $\mathbf{9}-\mathrm{HCN} \mathbf{6 1}$, HCN 24 - dimethylamine 83, HCN 24 - dimethyl ether 63, HCN 24 - dimethyl sulfide 56, HCN 24 - furan 45, HCN 24 HCN 61, HCN 24 - methylamine 91, HCN 24 - methanol 71, HCN 24 - methanethiol53, HCN 24 - water 75, methanol 16 HCN 61, methanol 16 - methylamine 91, methanol 16 - methanol 71, methanol 16 - methanethiol 53, methanol 16 - water 75, pyrrole 21 - HCN 61, pyrrole 21 - water 75, methanethiol 3 - HCN 61, methanethiol 3 - methylamine 91, methanethiol 3 methanethiol 53, methanethiol 3 - water $\mathbf{7 5}$, water 15 - dimethylamine 83, water $\mathbf{1 5}$ - dimethyl ether $\mathbf{6 3}$, water $\mathbf{1 5}$ - dimethyl sulfide 56, water 15 - furan $\mathbf{4 5}$, water 15 - HCN 61, water 15 - methylamine $\mathbf{9 1}$, water 15 - methanol 71, water 15 - pyridine 82, water $\mathbf{1 5}$ - methanethiol 53, water $\mathbf{1 5}$ - trimethylamine $\mathbf{7 8}$ and water $\mathbf{1 5}$ - water $\mathbf{7 5}$. Optimizations were performed with the counterpoise correction. The energy difference between B3LYP/cc-pVDZ and MP2/aug-cc-pVTZ is systematic and within about $1.0 \mathrm{kcal} / \mathrm{mol}$, (Fig. S3), in agreeement with previous studies. ${ }^{80}$ The overall trend was preserved (Pearson correlation coefficient: 0.88 ) indicating a satisfactory agreement between the two methods.

As a validation, we tested that the relationship of $D_{[H F]}$ with strength for thioketone $57\left(\Delta E_{o p t}=-7.36\right)$ and acetone $\mathbf{6 7}$ $\left(\Delta E_{\text {opt }}=-10.11\right)$ was consistent between results obtained with B3LYP and MP2. The values of $\varepsilon_{[H F]}$ calculated with MP2 $(-3.7$ and $-2.9 \mathrm{kcal} / \mathrm{mol})$ showed to be very close to those calculated with B3LYP $(-3.49 \mathrm{kcal} / \mathrm{mol}$ and $-2.56 \mathrm{kcal} / \mathrm{mol})$.

Accuracy of anisotropy profiles. To assess the quality of directionality profiles calculated with B3LYP/cc-pVDZ we used MP2/aug-cc-pVTZ to compute domes for donor and acceptor sites of water $(\mathbf{1 5}, \mathbf{7 5})$, imidazole $(\mathbf{2 5}, \mathbf{9 2})$, methyl-acetamide $(\mathbf{1 8}, \mathbf{9 0})$, methyl-thioacetamide (not numbered as donor; compound 62 as acceptor), phenol (26, 55), and TMAO (97). Results are summarized in Supplementary Table S2. For acceptors, the equal area projections obtained with the two levels of theory are nearly indistinguishable. The largest numerical deviation of $D_{[H F]}$ is observed for phenol (MP2: 0.37; B3LYP: 0.42). This is 


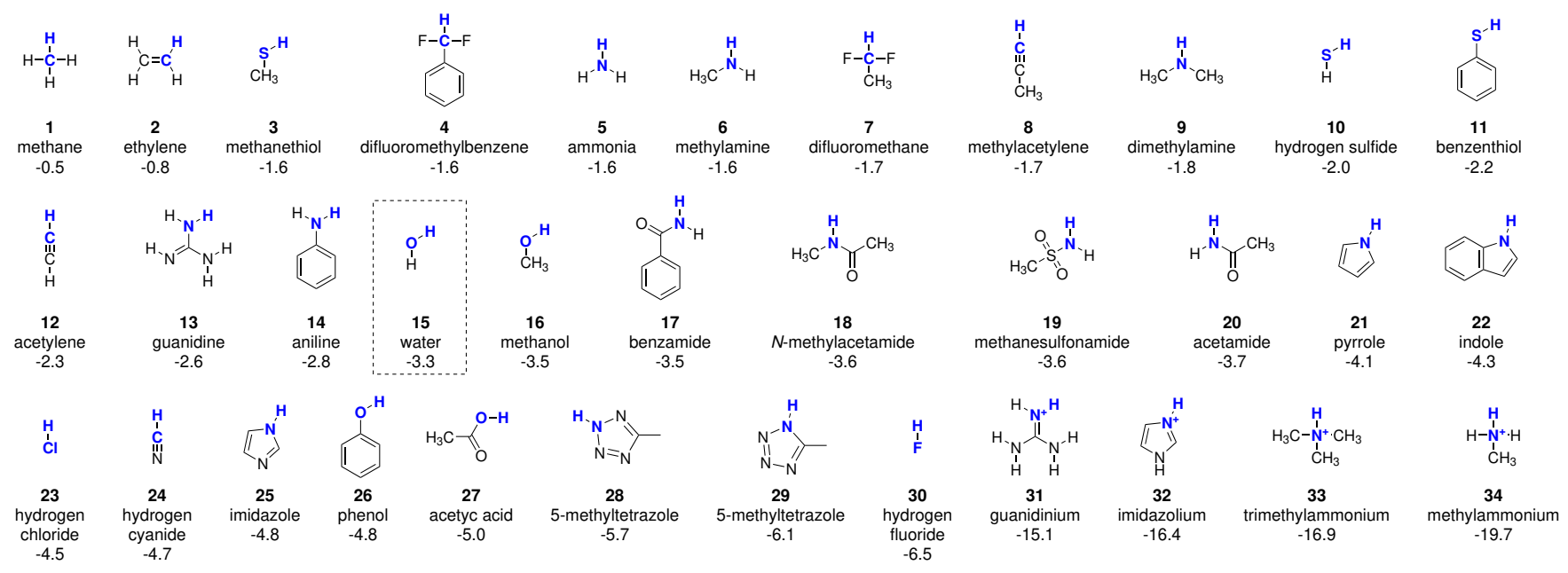

Supplementary Figure S1 Donor groups ranked by their interaction energy (in kcal/mol, below each molecule) with the HCN acceptor probe 61 . Donor target atoms are colored in blue

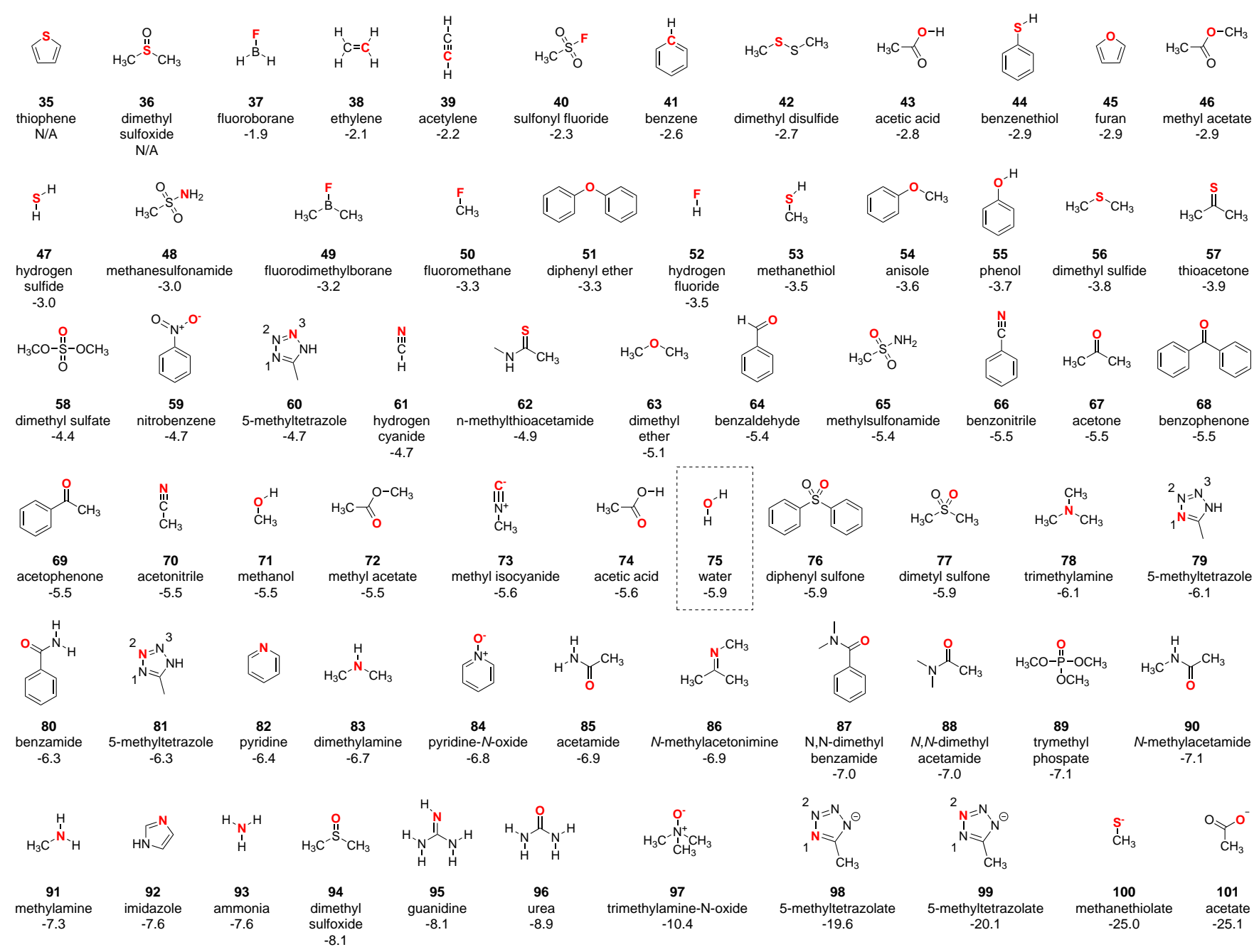

Supplementary Figure S2 Acceptor groups ranked by their interaction energy (in kcal/mol, below each molecule) with the HCN donor probe 24 . Acceptor target atoms are colored in red 


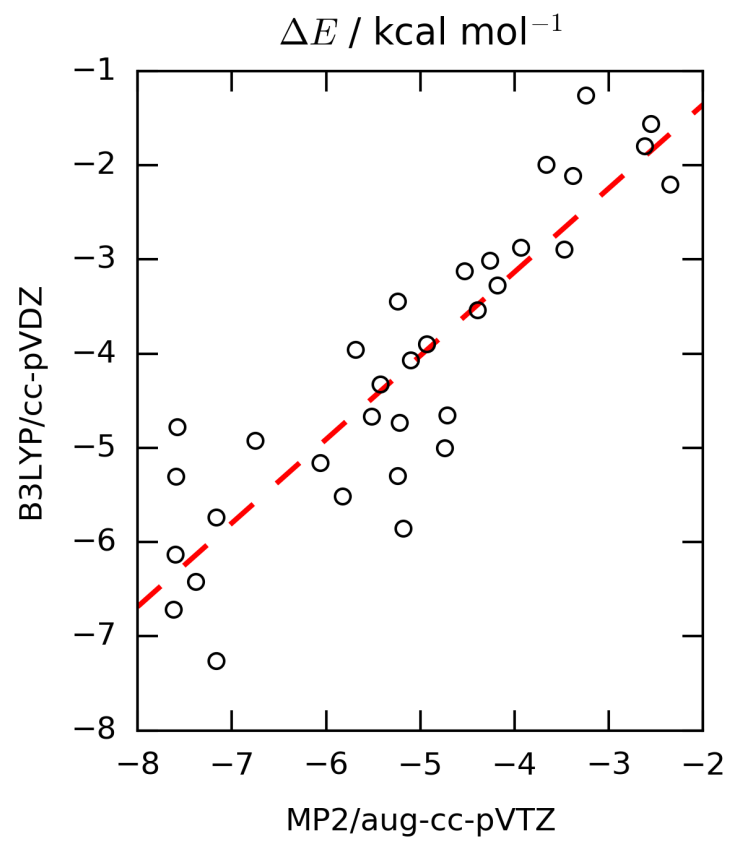

Supplementary Figure S3 Interaction energies of 33 dimers at the MP2/aug-cc-pVTZ and B3LYP/cc-pVDZ levels of theory. Each dimer was fully optimized with both levels of theory using the counterpoise correction. The red dashed line represents a regression model for this set of dimers.

likely due to better characterization of the delocalized electrons in the aromatic compound with MP2, while, visual inspection of the equal area projections revealed practically identical qualitative profiles (Fig. S4).

With respect to donor directionality, MP2/aug-cc-pVTZ results showed systematic profile broadening for imidazole, Nmethylacetamide and $\mathrm{N}$-methylthioacetamide, and to a lesser extent for water and phenol (Fig. S5). This was attributed to underestimation of the magnitude of dispersion interactions by B3LYP ${ }^{87}$. Nevertheless, visual inspection of the domes shows very similar anisotropy maps. In summary, the confidence on $D_{[\text {probe }]}$ values calculated with B3LYP is higher for acceptor sites than for donors. This is probably due to the use of hydrogen fluoride (HF) to probe acceptors. HF has only 10 electrons and is highly polarized, minimizing the relative contribution of dispersion to the overall interaction energy. Overall, there is excellent agreement between B3LYP/cc-pVDZ and MP2/aug-cc-pVTZ for acceptor domes, as long as the probe is HF.

The B3LYP density function is adequate for the study of anisotropy in cooperative HBs (Supplementary Fig. S18), because three-body interaction energies are well described, even without dispersion corrections ${ }^{88,89}$.

Supplementary Table S2 Directionality indexes calculated with MP2/aug-cc-pVTZ and B3LYP/cc-pVDZ. HF probes acceptor sites. HCN probes donor sites

\begin{tabular}{rrrrrrr} 
& & & \multicolumn{2}{c}{ MP2/aug-cc-PVTZ } & \multicolumn{2}{c}{ B3LYP/cc-pVDZ } \\
\cline { 5 - 7 } target type & probe & target & $\Delta E_{\text {opt }}^{\prime}($ probe $)$ & $D_{[\text {probe }]}$ & $\Delta E_{\text {opt }}^{\prime}($ probe $)$ & $D_{[\text {probe }]}$ \\
\hline acceptor & HF & water 75 & -8.0 & 0.34 & -8.6 & 0.34 \\
acceptor & HF & phenol 55 & -7.3 & 0.37 & -6.2 & 0.42 \\
acceptor & HF & imidazole 92 & -13.4 & 0.43 & -11.6 & 0.45 \\
acceptor & HF & $N$-methyl-acetamide 90 & -11.4 & 0.21 & -10.6 & 0.21 \\
acceptor & HF & $N$-methyl-thioacetamide 62 & -9.4 & 0.38 & -8.0 & 0.36 \\
acceptor & HF & TMAO 97 & -20.0 & 0.25 & -18.3 & 0.25 \\
\hline donor & HCN & water 15 & -4.2 & 0.48 & -3.2 & 0.47 \\
donor & HCN & phenol 26 & -6.1 & 0.47 & -4.8 & 0.53 \\
donor & HCN & imidazole 25 & -5.9 & 0.32 & -4.8 & 0.43 \\
donor & HCN & $N$-methyl-acetamide 18 & -5.0 & 0.35 & -3.6 & 0.46 \\
donor & HCN & $N$-methyl-thioacetamide & -5.8 & 0.33 & -4.4 & 0.43 \\
\hline
\end{tabular}

Internal geometry constraints. When calculating domes, we generated input geometries in an automated fashion and performed a single point energy evaluations for each geometry. During these calculations structures were not allowed to relax, specifically bond lenghts and bond angles, in order to preserve the intended $(r, \phi, \theta)$ values. This could create artificially high energies when angles are far from the optimum, and repulsions occur. To assess the effect of these restraints on calculated energies, we performed a relaxed energy scan along the $\phi$ angle of the HF-pyridine dimer (Fig. S6, black circles), and compared it with the energy profile obtained using rigid monomers at fixed distance (Fig. S6, red dashed line). The distance between the red dashed line and the black circles is the energy error in the domes if fixed $r$ were used. When the distance between the donor 

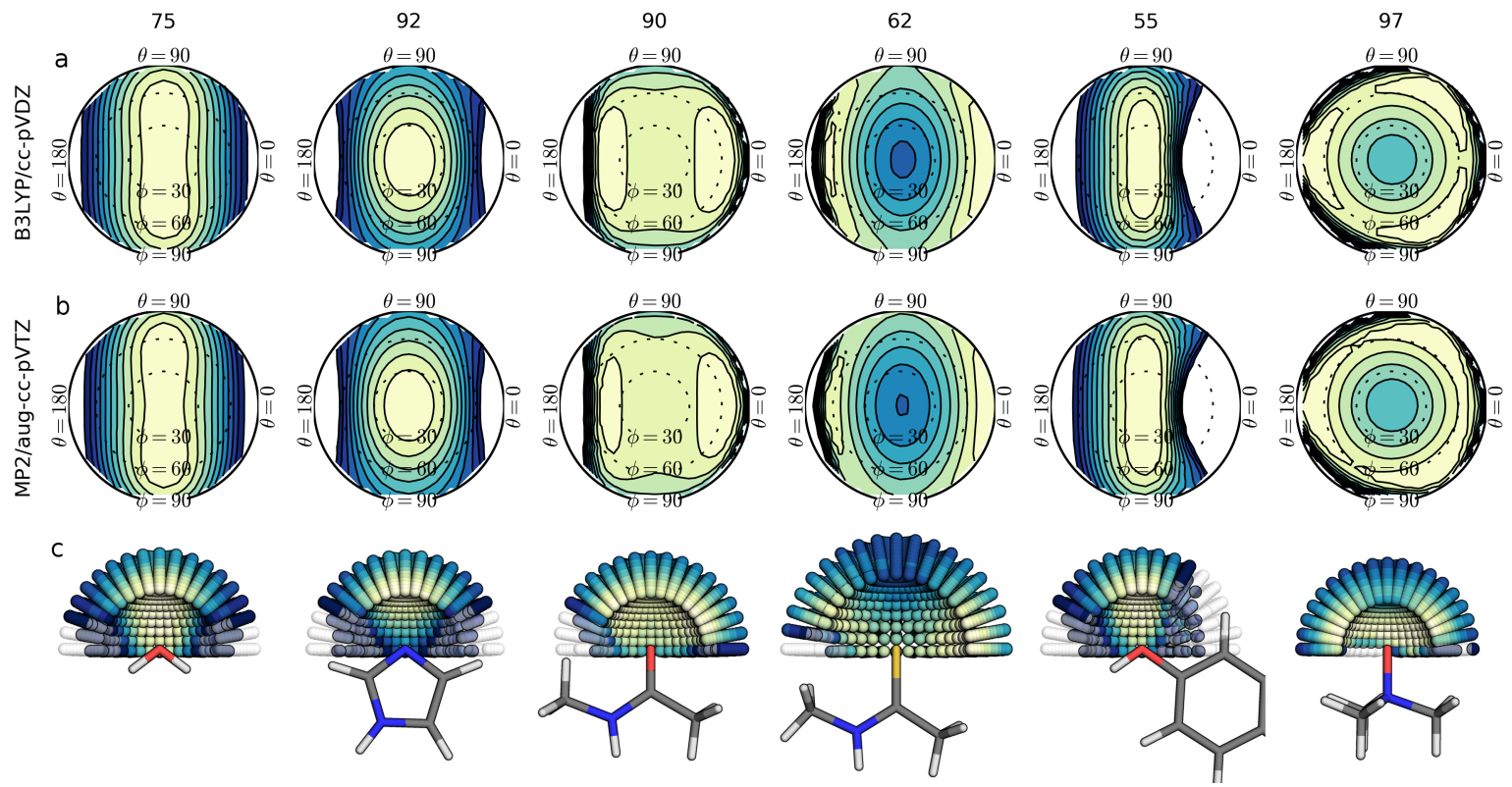

Supplementary Figure S4 Directionality profiles of acceptor sites calculated with B3LYP/cc-pVDZ (a) and MP2/aug-cc-pVTZ (b and c).
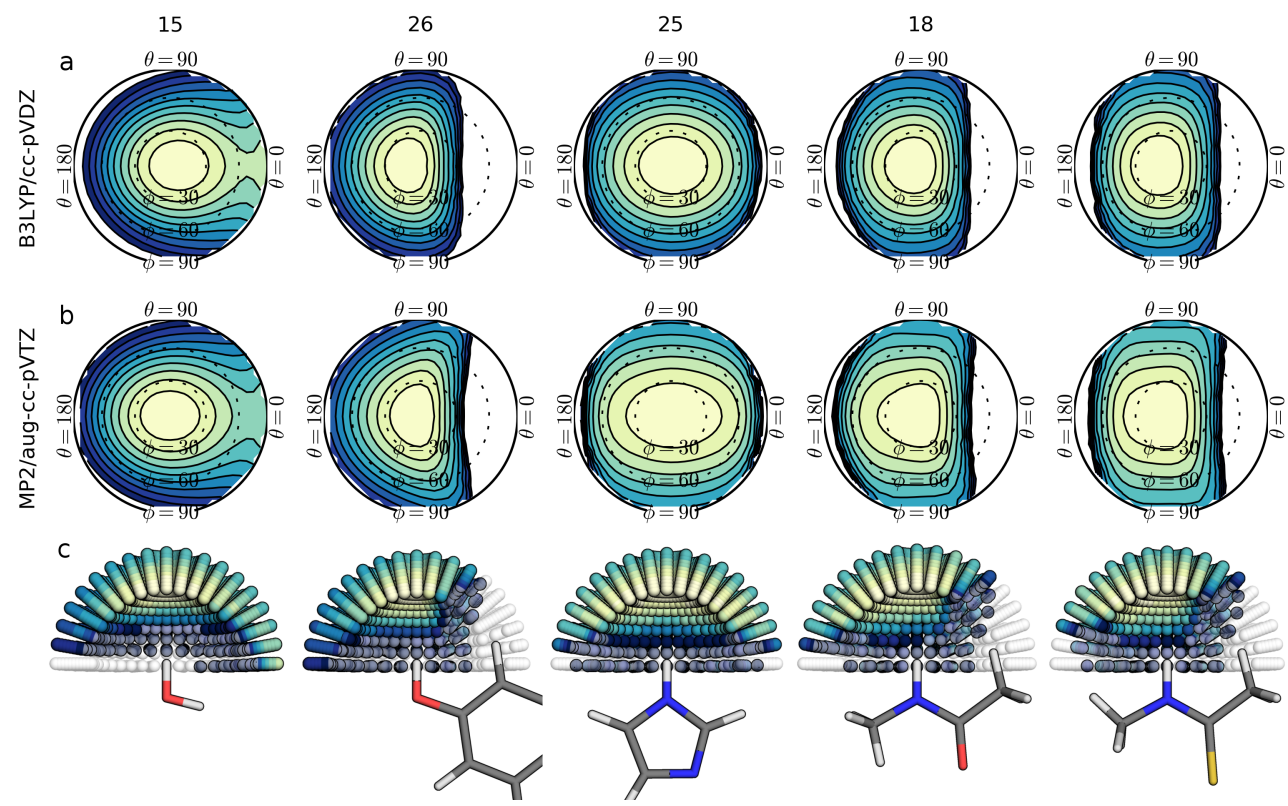

Supplementary Figure S5 Directionality profiles of donor sites calculated with B3LYP/cc-pVDZ (a) and MP2/aug-cc-pVTZ (b and c). 


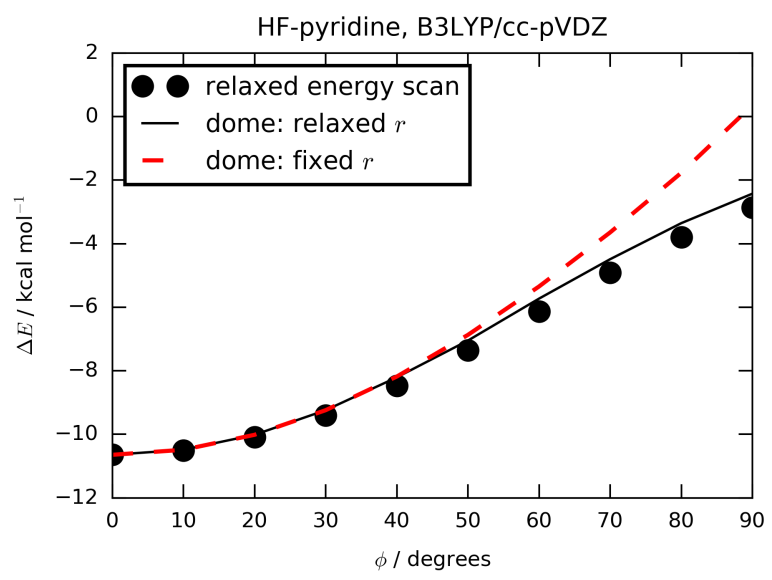

Supplementary Figure S6 Directionality profiles obtained using the rigid monomer approach compared to a relaxed energy scan (in which bond lengths and angles are allowed to relax).

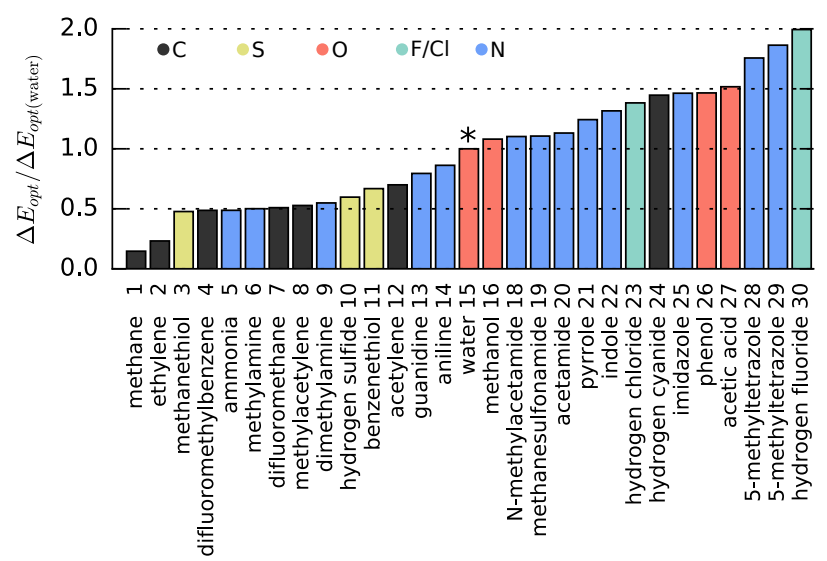

Supplementary Figure $\mathbf{S 7}$ Comparison of donor strengths $\Delta E_{\text {opt }}$ ranked by their strength relative to water, and colored by element type. Values are calculated with $\mathrm{HCN}$ acceptor probe 61.

hydrogen and the acceptor atom is allowed to relax (Fig. S6, black solid line), negligible differences are found. Therefore the $r$ distance scan was included in the calculation of domes.

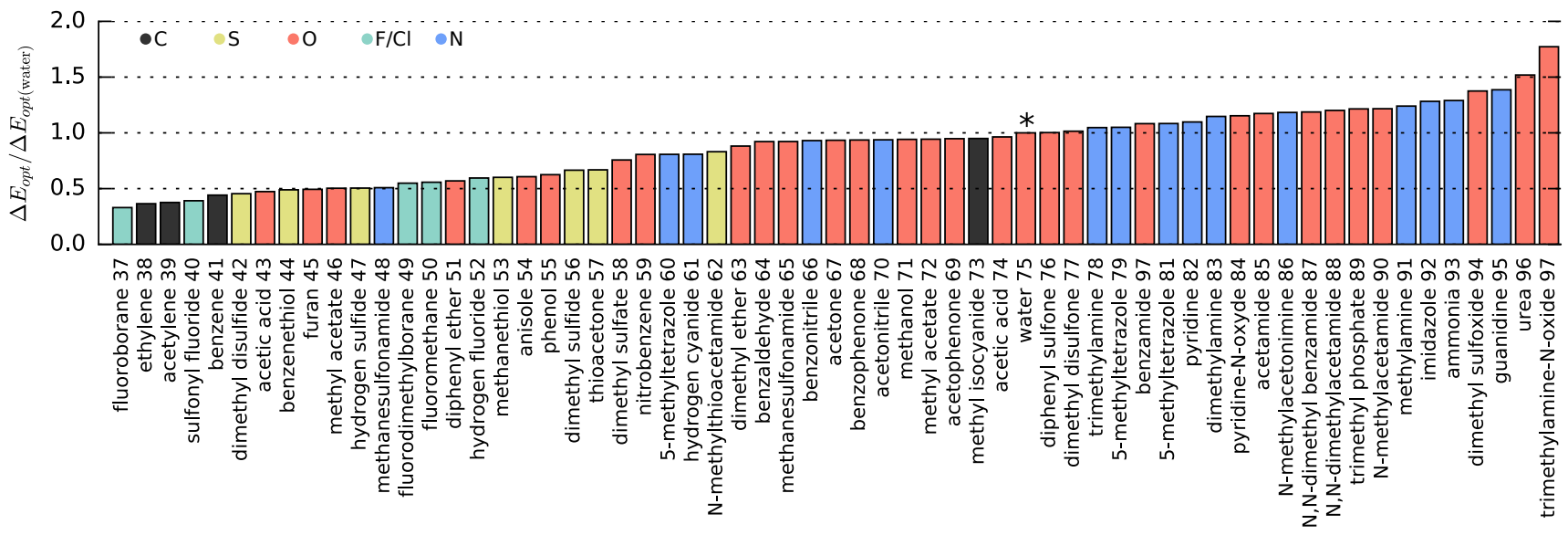

Supplementary Figure $\mathbf{S} 8$ Comparison of acceptor strengths $\Delta E_{\text {opt }}$ ranked by their strength relative to water, and colored by element type. Values are calculated with $\mathrm{HCN}$ donor probe 24. 


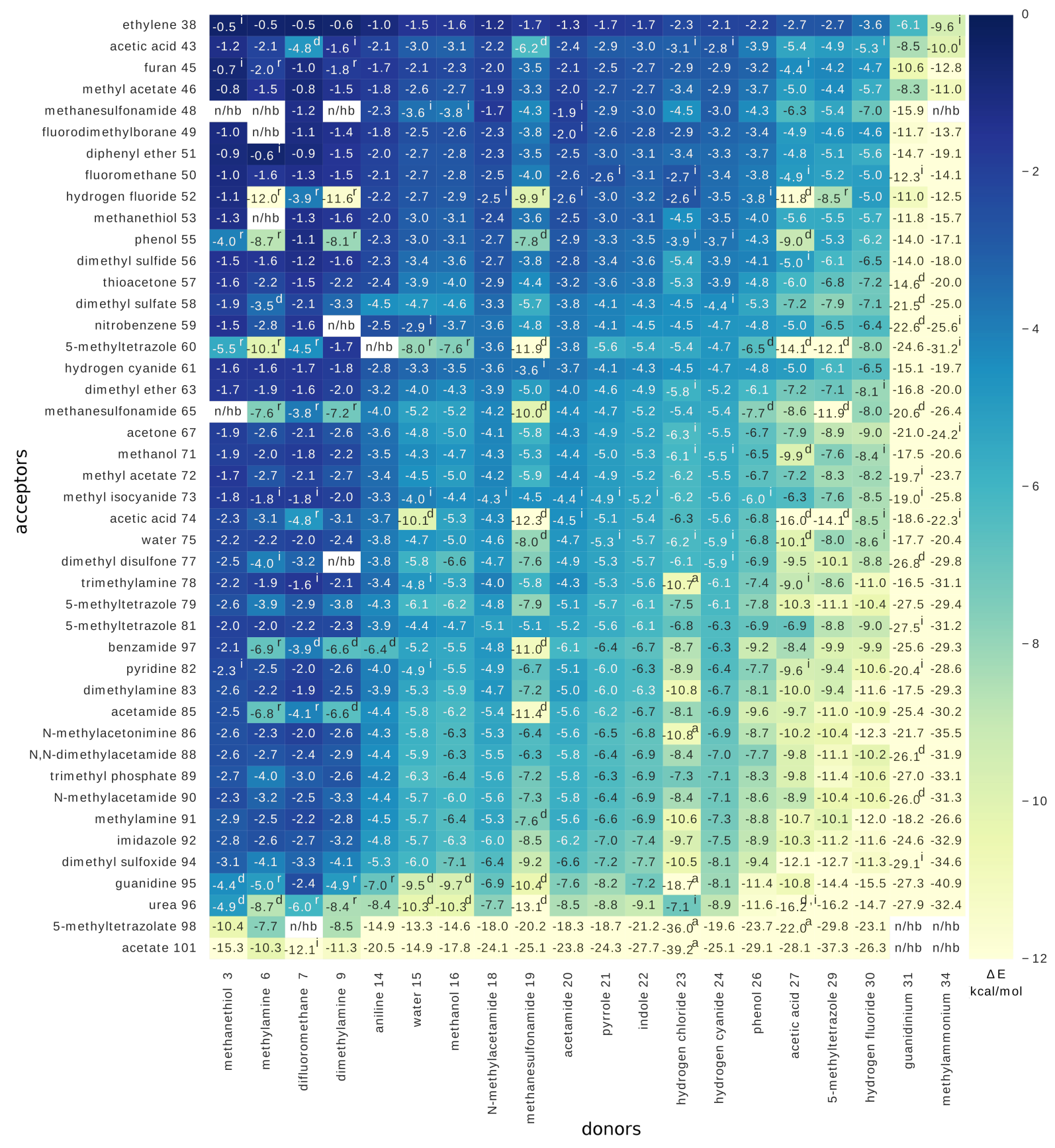

Supplementary Figure S9 Interaction energies between donor (columns) and acceptor (rows) groups, ranked by their interaction energy with the reference probe $\mathrm{HCN}$ ( 61 and 24, respectively). Annotations: $i$ imaginary frequencies (optimized to transition state); $d$ multi-furcated HB; $r$ inversion of donor/acceptor roles; a dissociation of acidic proton; $n / h b$ not a hydrogen bond.

Supplementary Table S3 Directionality and strength in cooperative HBs: acceptor sites.

\begin{tabular}{|c|cc|cc|}
\hline & \multicolumn{2}{|c|}{ In vacuo } & \multicolumn{2}{c|}{ Cooperative } \\
\hline Target & $\Delta E_{\mathrm{opt}}[\mathrm{HF}]$ & $D_{[\mathrm{HF}]}$ & $\Delta E_{\mathrm{wat}[\mathrm{HF}]}$ & $D_{[\mathrm{HF}]}$ \\
\hline water 75 & -8.7 & 0.34 & -10.9 & 0.31 \\
N-methylacetamide 90 & -10.4 & 0.21 & -10.9 & 0.21 \\
imidazole 92 & -11.6 & 0.45 & -13.4 & 0.42 \\
\hline
\end{tabular}

Supplementary Table S4 Directionality and strength in cooperative HBs: donor sites.

\begin{tabular}{|c|cc|cc|}
\hline & \multicolumn{2}{|c|}{ In vacuo } & \multicolumn{2}{c|}{ Cooperative } \\
\hline Target & $\Delta E_{\text {opt }[\mathrm{HCN}]}$ & $D_{[\mathrm{HCN}]}$ & $\Delta E_{\text {wat }[\mathrm{HCN}]}$ & $D_{[\mathrm{HCN}]}$ \\
\hline water 15 & -3.2 & 0.47 & -3.9 & 0.48 \\
N-methylacetamide 18 & -3.6 & 0.46 & -3.7 & 0.47 \\
imidazole 25 & -4.8 & 0.43 & -5.2 & 0.43 \\
\hline
\end{tabular}




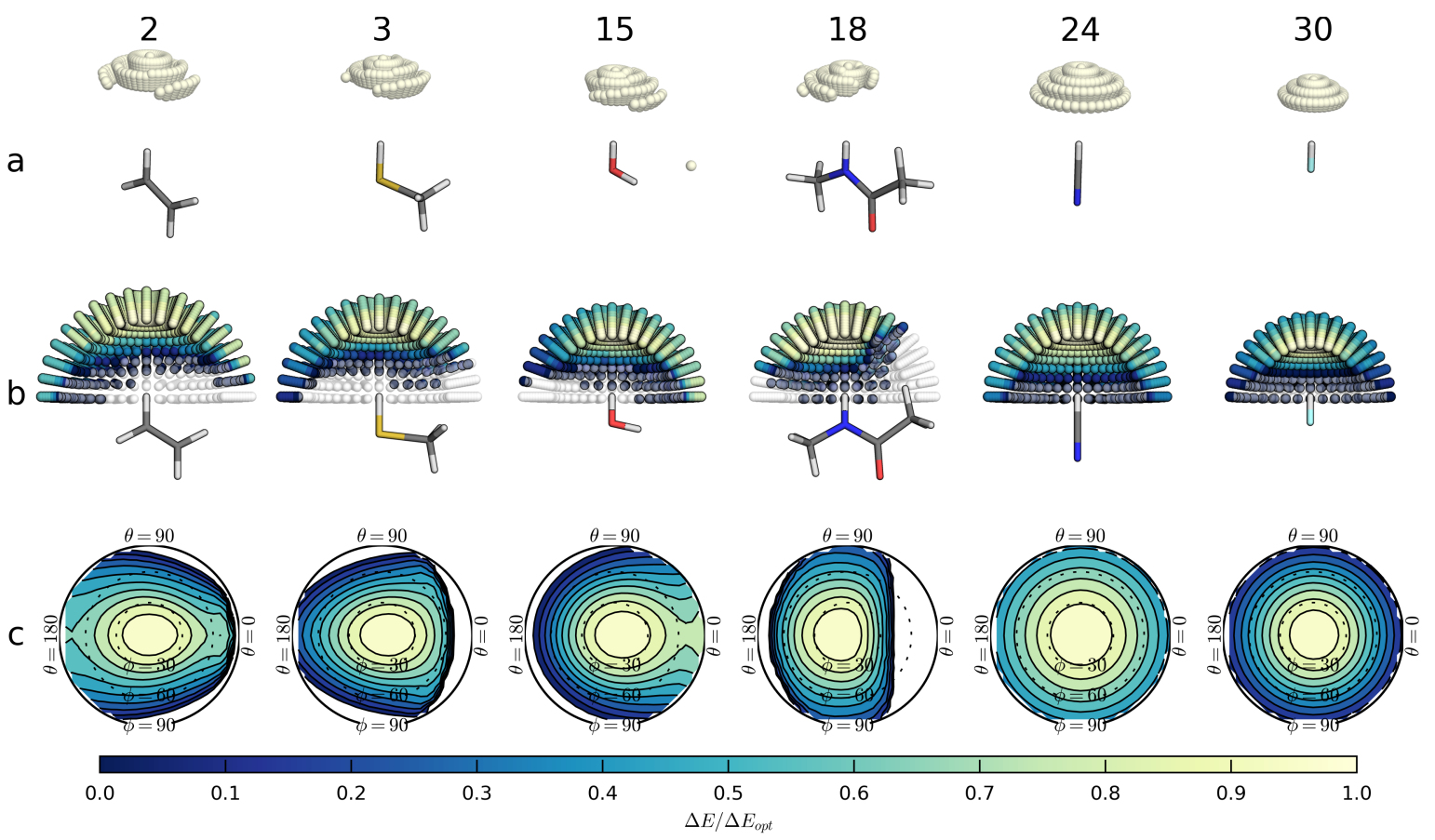

Supplementary Figure S10 Directionality of donors. $D_{[H C N]}$ values range from 0.45 to 0.54 , except for $\mathrm{HCN} 24\left(D_{[H C N]}=0.36\right)$.

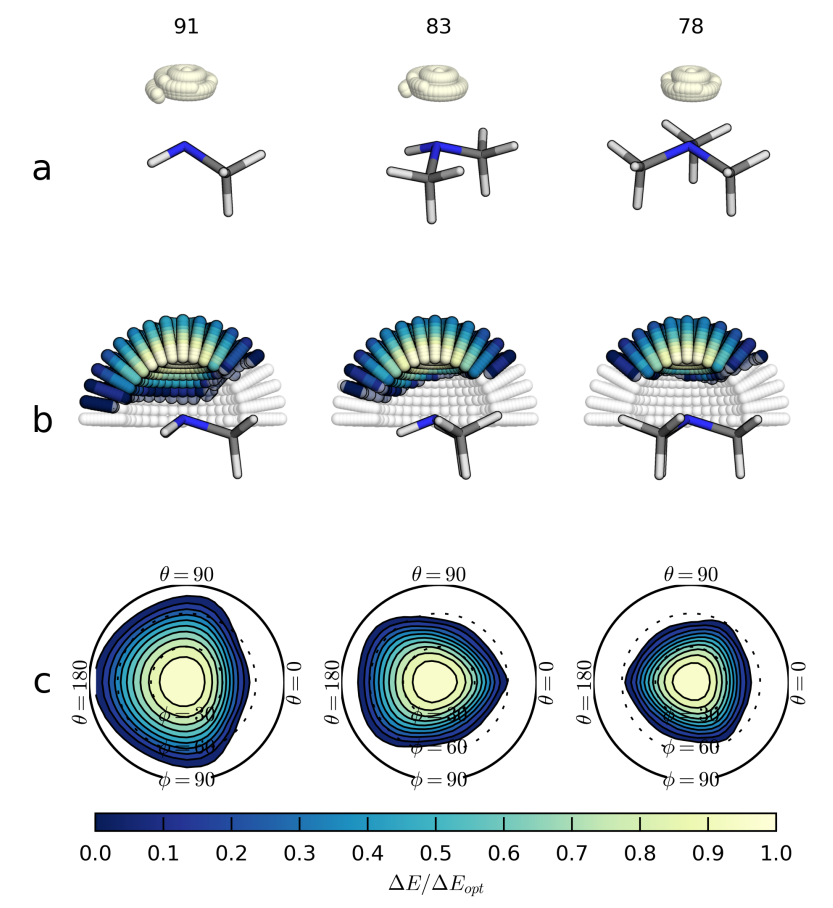

Supplementary Figure S11 Directionality of amine series: methylamine 93, dimethylamine 83 and trimethylamine $\mathbf{7 8 .}$ 


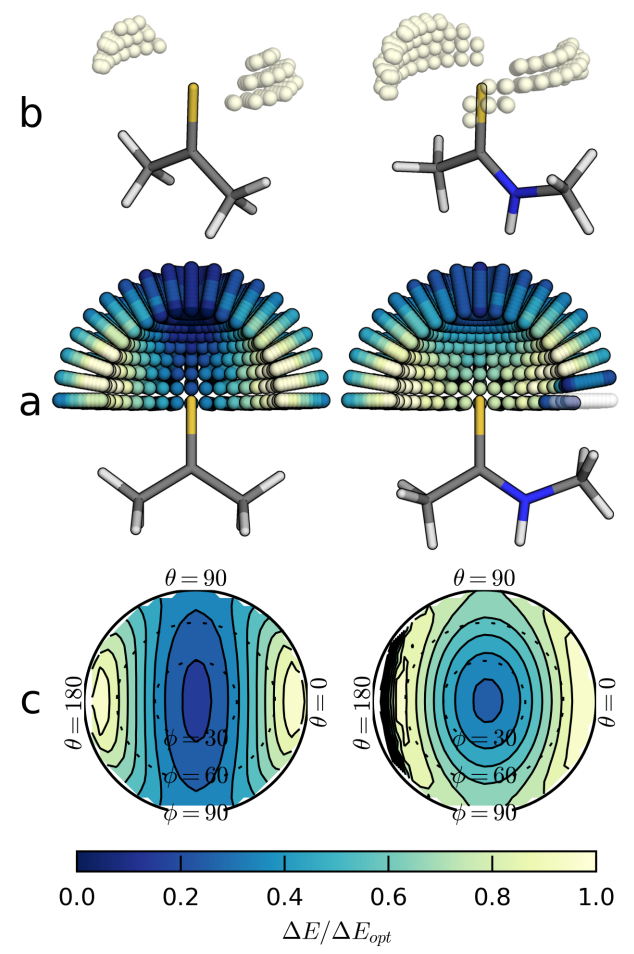

Supplementary Figure S12 Directionality of $s p^{2}$ sulfur: thioacetone 57 and $\mathrm{N}$-methyl thioacetamide 62.

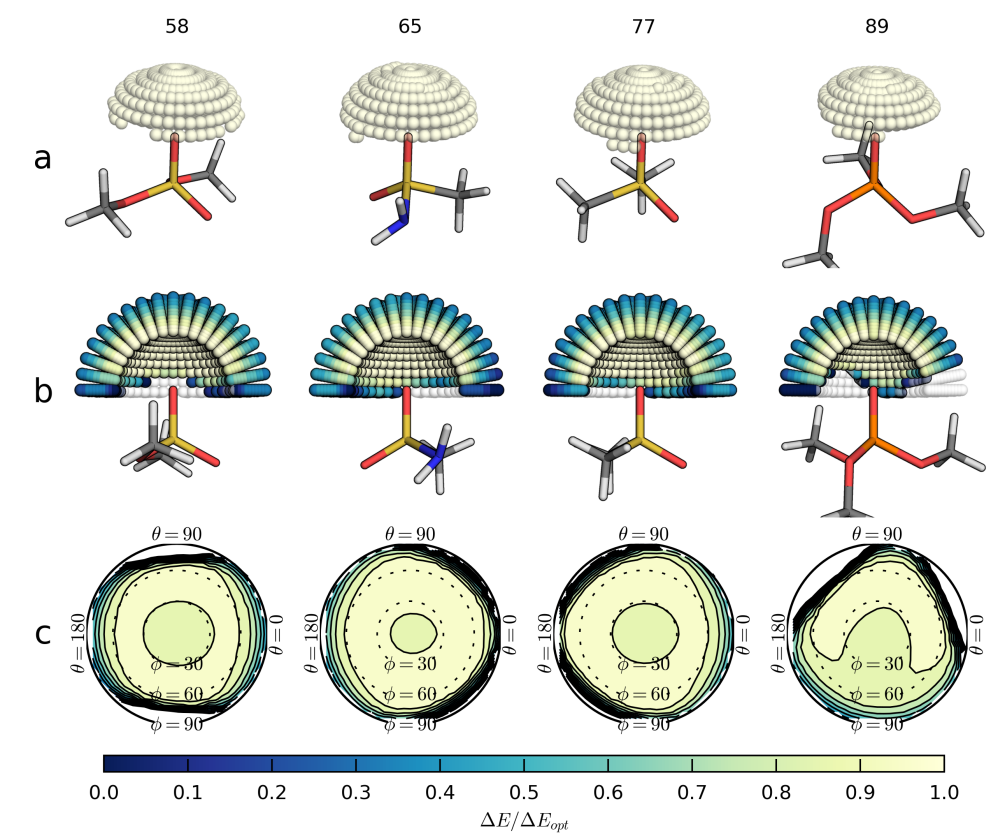

Supplementary Figure S13 Directionality of oxygen bound to sulfur or phosphate: dimethyl sulfate $\mathbf{5 8}$, methylsulfonamide $\mathbf{6 5}$, dimethyl sulfone $\mathbf{7 7}$, and trimethyl phosphate 89. 


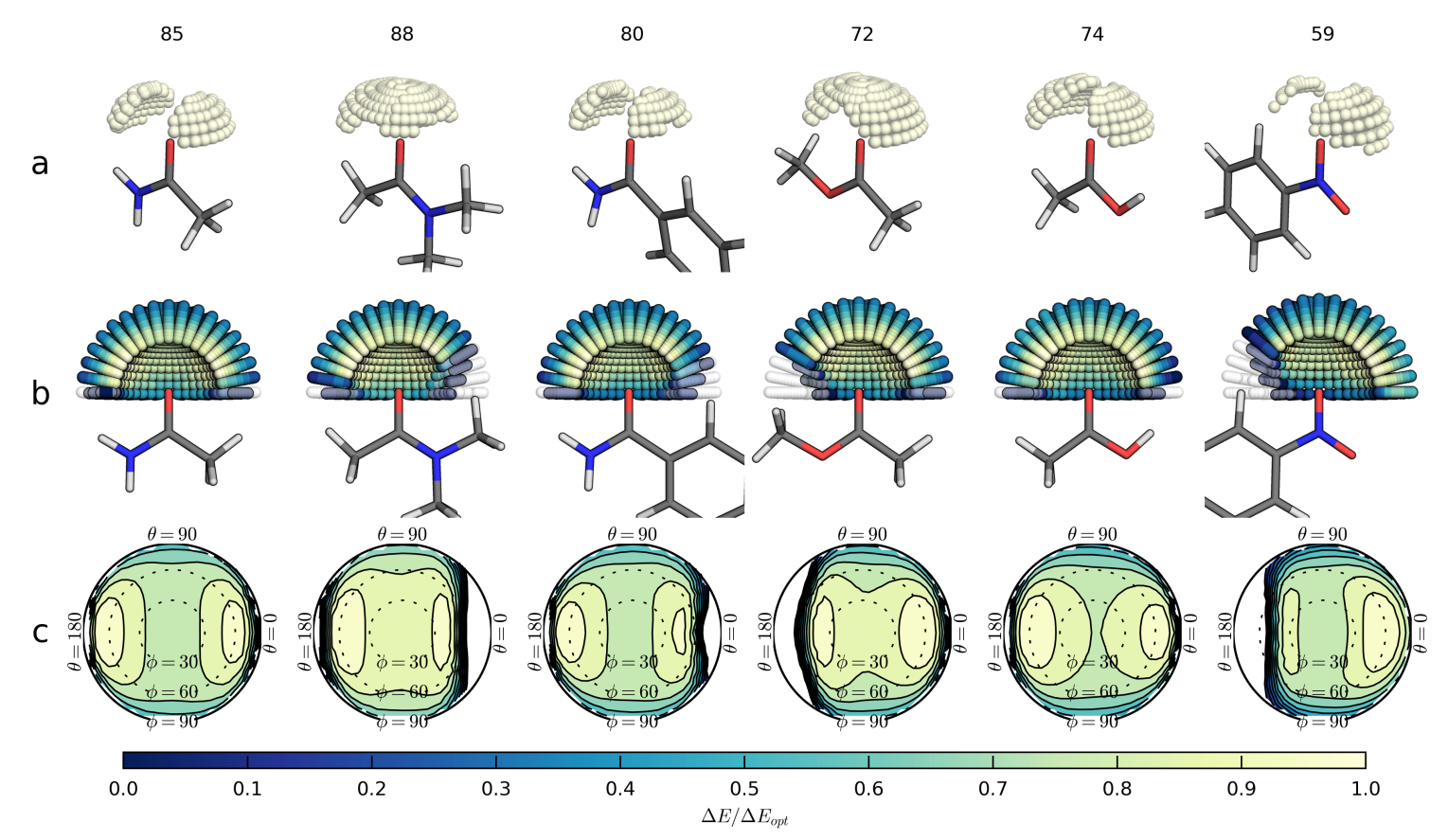

Supplementary Figure $\mathbf{S} 14$ Directionality of various $s p^{2}$ oxygens: acetamide $\mathbf{8 5}, \mathrm{N}, \mathrm{N}$-dimethyl acetamide $\mathbf{8 8}$, benzamide $\mathbf{8 0}$, methyl acetate $\mathbf{7 2}$, acetic acid $\mathbf{7 4}$, and nitrobenzene 59.
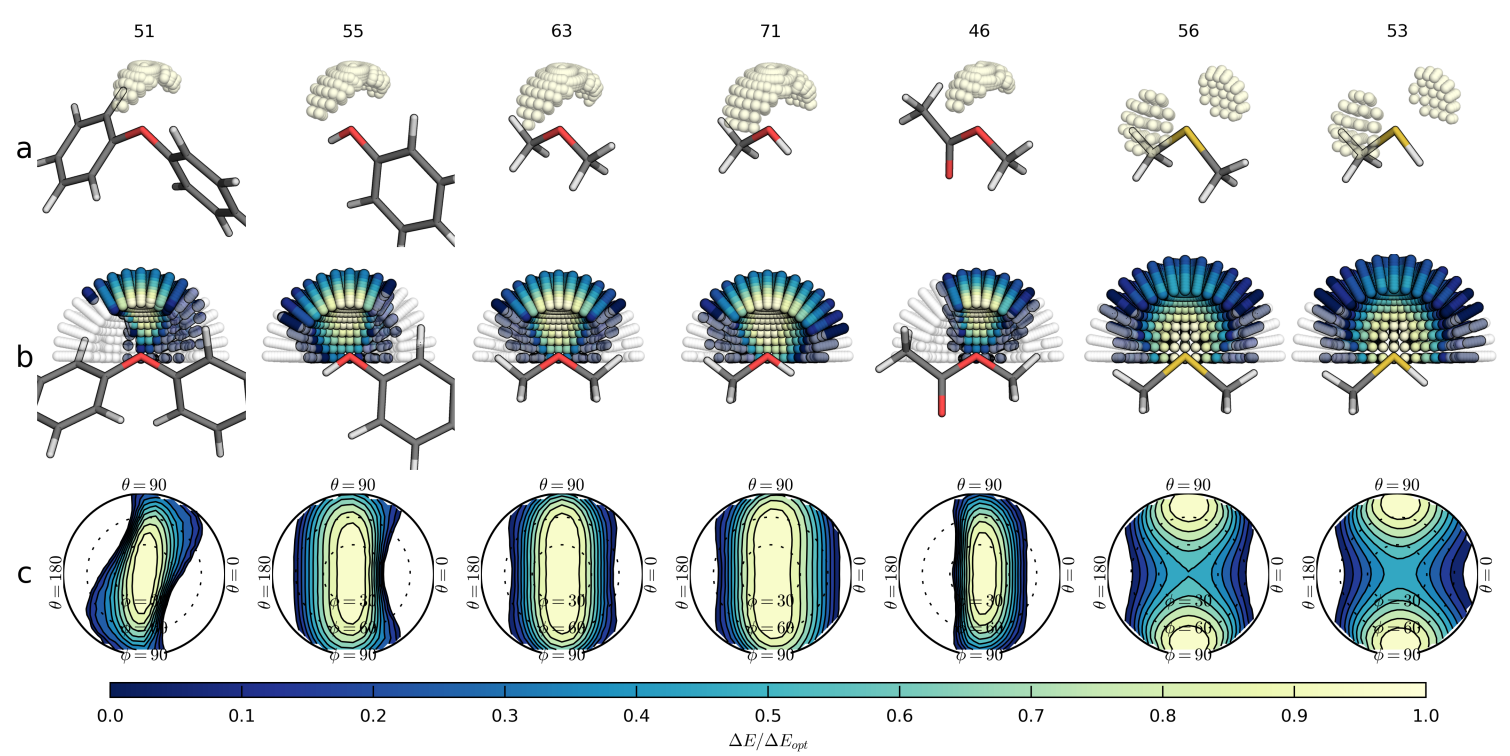

Supplementary Figure S15 Directionality of various $s p^{3}$ oxygen and sulfur groupss: diphenyl ether $\mathbf{5 1}$, phenol $\mathbf{5 5}$, dimethyl ether $\mathbf{6 3}$, methanol $\mathbf{7 1}$, methyl acetate $\mathbf{4 6}$, dimethyl sulfide $\mathbf{5 6}$, and methanethiol $\mathbf{5 3}$. 
a<smiles>[Li]</smiles>

b
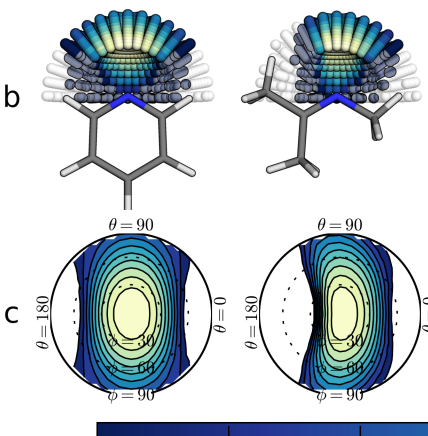

0.0

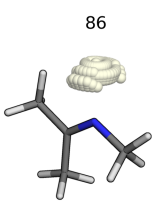

95

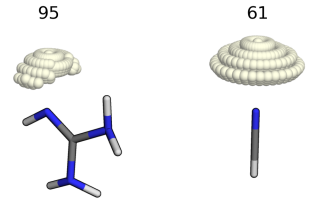

entrm

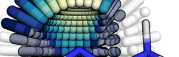

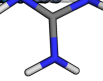
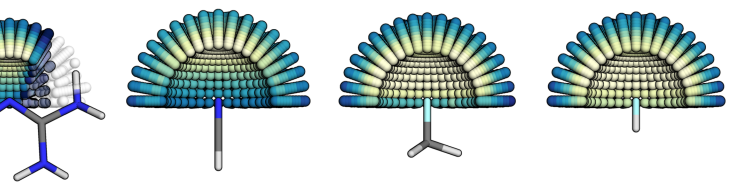

b
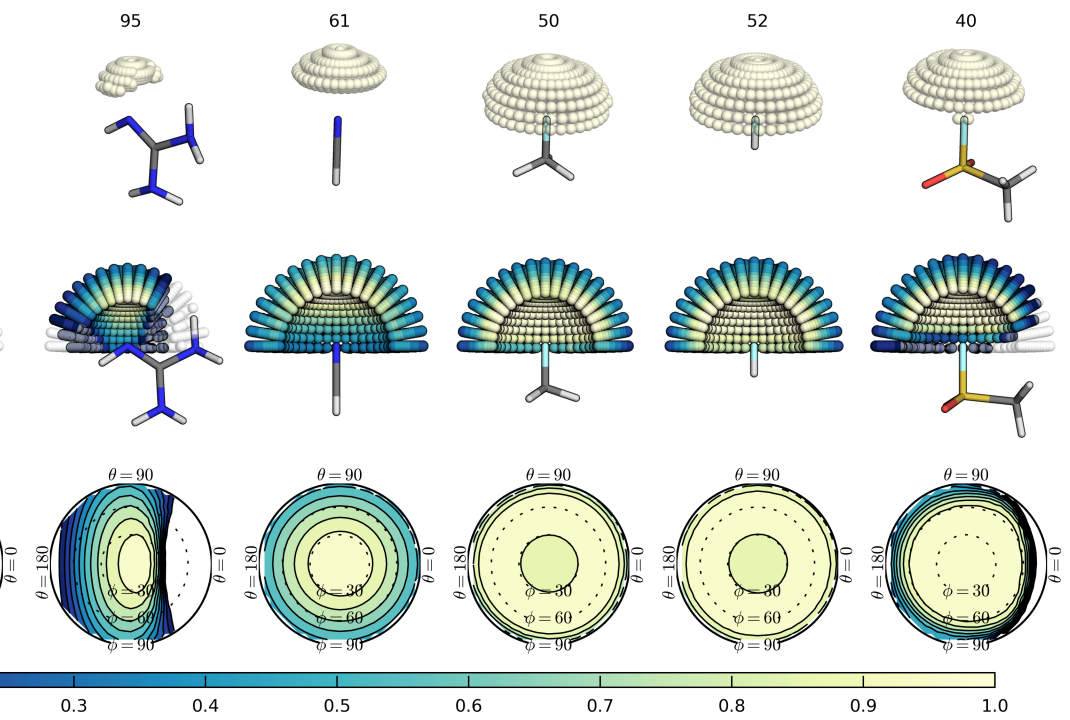

Supplementary Figure S16 Directionality of nitrogen (pyridine 82, N-methylacetoimine 86, guanidine 95, HCN 61) and fluorine (fluoromethyl 50, HF 52, sulfonyl fluoride 40) acceptors. 

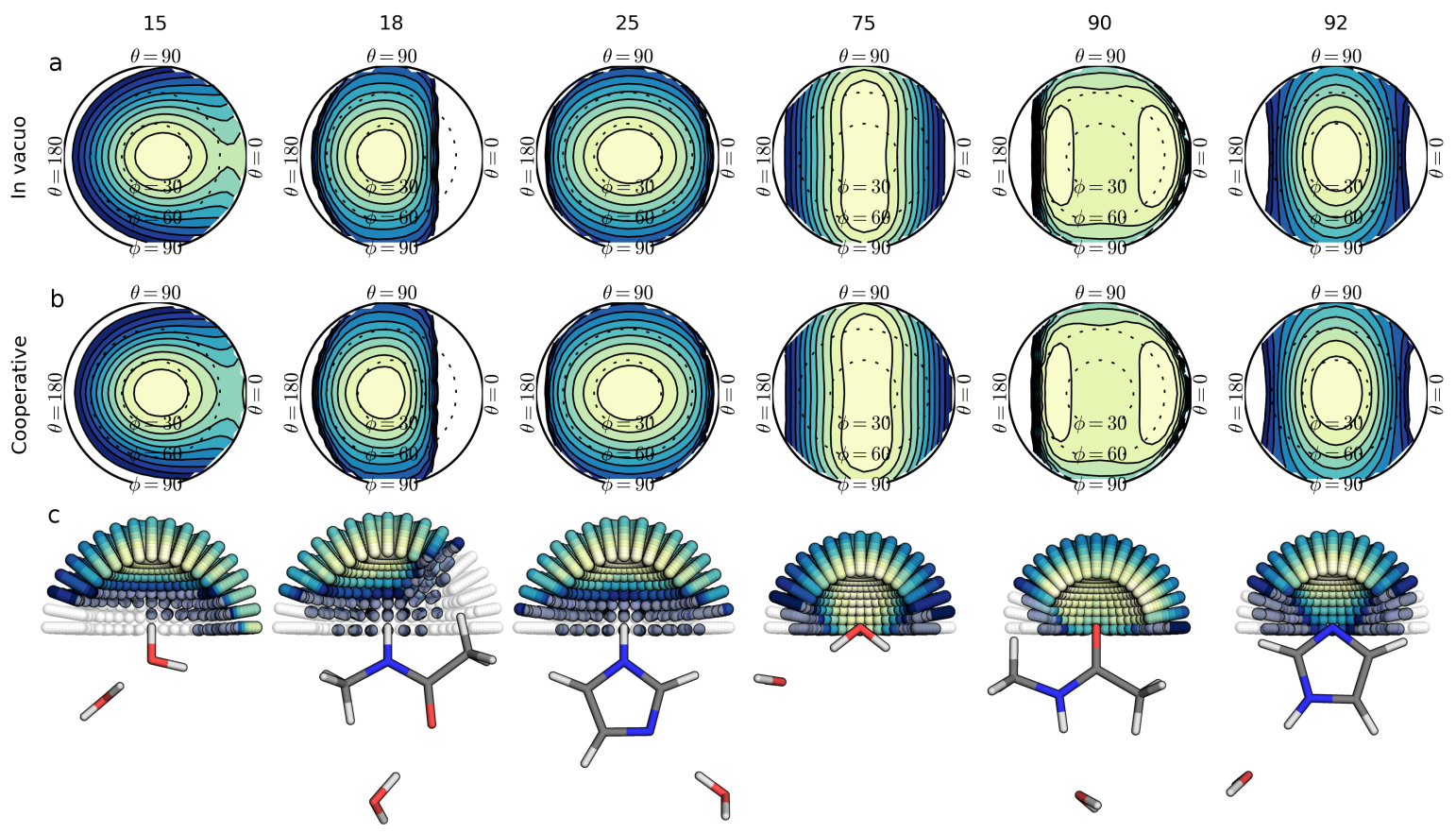

Supplementary Figure S18 Directionality profiles with (b) and without (a) cooperative effects. An explicit water molecule was added to create a HB network involving the added water molecule, the target molecule under study, and the probe. 